\title{
An Algebraic Exploration of Dominating Sets and Vizing's Conjecture
}

\author{
S. Margulies \\ Department of Mathematics \\ Pennsylvania State University \\ State College, PA \\ margulies@math.psu.edu
}

\author{
I. V. Hicks \\ Computational and Applied Mathematics \\ Rice University \\ Houston, TX \\ ivhicks@rice.edu
}

Submitted: Oct 7, 2011; Accepted: Mar 29, 2012; Published: Apr 7, 2012

Mathematics Subject Classifications: 05C69, 13P10

\begin{abstract}
Systems of polynomial equations are commonly used to model combinatorial problems such as independent set, graph coloring, Hamiltonian path, and others. We formulate the dominating set problem as a system of polynomial equations in two different ways: first, as a single, high-degree polynomial, and second as a collection of polynomials based on the complements of domination-critical graphs. We then provide a sufficient criterion for demonstrating that a particular ideal representation is already the universal Gröbner bases of an ideal, and show that the second representation of the dominating set ideal in terms of domination-critical graphs is the universal Gröbner basis for that ideal. We also present the first algebraic formulation of Vizing's conjecture, and discuss the theoretical and computational ramifications to this conjecture when using either of the two dominating set representations described above.
\end{abstract}

Keywords: dominating sets, Vizing's conjecture, universal Gröbner bases

\section{Introduction}

The combination of non-linear models and techniques from computer algebra is gaining acceptance as a tool for exposing combinatorial properties of graph-theoretic problems. For example, prior work on polynomial encodings includes colorings $[3,13,18,21,23$, 24, 25, 27, 28, 30], stable sets [20, 21, 25, 31], matchings [14], and flows [3, 29, 30]. These non-linear encodings have been used to prove combinatorial results [2, 22, 24], and Gröbner bases have been the building blocks of algorithms for solving integer programs $[6,10,34]$, but techniques from computer algebra are not widely used by combinatorists and graph theorists, and thus have not yet been deeply explored. In this paper, we move 
beyond simply formulating a graph-theoretic problem as a system of polynomial equations, and instead formulate an entire graph-theoretic conjecture using systems of polynomial equations and algebraic techniques. Although the method we introduce here can probably be applied to other open questions involving inequalities, we focus on the dominating set problem and an algebraic approach to a famous open question from domination theory: Vizing's conjecture.

Given a graph $G$, a set $D \subseteq V(G)$ is a dominating set if for all $v \notin D$, there is a $u \in D$ such that $v$ is adjacent to $u$ in $G$. The domination number of $G$, denoted by $\gamma(G)$, is the size of a minimum dominating set in $G$. The decision problem of determining whether a given graph has a dominating set of size $k$ is NP-complete [15]. Given graphs $G$ and $H$, the Cartesian product graph $G \square H$ has vertex set $V(G) \times V(H)$, and edge set

$$
E(G \square H)=\left\{\left(g h, g^{\prime} h^{\prime}\right): g=g^{\prime} \text { and }\left(h, h^{\prime}\right) \in E(H) \text {, or } h=h^{\prime} \text { and }\left(g, g^{\prime}\right) \in E(G)\right\} \text {, }
$$

where $g, g^{\prime} \in V(G)$ and $h, h^{\prime} \in V(H)$. In 1968, V. Vizing conjectured a beautiful relationship between domination numbers and Cartesian product graphs:

Conjecture 1 (Vizing [35], 1968). Given graphs $G$ and $H, \gamma(G) \gamma(H) \leqslant \gamma(G \square H)$.

Vizing's conjecture is an active area of research spanning over forty years. Early results have focused on proving the conjecture holds for a certain classes of graphs. For example, in 1979, Barcalkin and German [5] proved that Vizing's conjecture holds for graphs satisfying a certain "partitioning condition" on the vertex set. The idea of a "partitioning condition" inspired work for the next several decades, as Vizing's conjecture was shown to hold on paths, trees, cycles, chordal graphs, graphs satisfying certain coloring properties, and graphs with $\gamma(G) \leqslant 2$. These results are clearly outlined in the 1998 survey paper by Hartnell and Rall [17]. In 2000, Clark and Suen [9] showed that $\gamma(G) \gamma(H) \leqslant 2 \gamma(G \square H)$, and in 2004, L. Sun [33] showed that Vizing's conjecture holds on graphs with $\gamma(G) \leqslant 3$. Finally, in 2009, Hartnell and Rall, et al. [7] contributed another thorough survey paper summarizing the work from 1968 to 2008, which contains new results, new proofs of existing results, and comments about minimal counter-examples.

Vizing's conjecture is of great interest to graph theorists for two primary reasons. First, it is an easily stated, easily understood combinatorial problem whose proof has eluded demonstration for decades. Second, it is of algorithmic interest, since domination in graphs has applications ranging from optimal fire station placement to wireless networks (see survey [7]), and additionally, product graphs are a common algorithmic tool for studying the scaling of NP-hard graph-theoretic invariants. In short, an elegant, elusive conjecture with algorithmic consequences will always excite exploration.

We begin by reviewing basic ideas from algebraic geometry: unions of varieties, intersections of ideals, notions of radical ideals, and universal Gröbner bases (Sections 2 and 3). In Section 3, for certain ideals, we develop a criterion for identifying a particular basis of the ideal as the universal Gröbner basis. In Section 4.1, we represent both the problem of finding graphs $G$ with dominating sets of size $k$, and the problem of finding graphs $G$ and $H$ with dominating sets of size $k, l$ respectively such that the Cartesian product graph $G \square H$ has a dominating set of size $r$, as systems of polynomial equations. In Section 4.2, 
we develop the idea of $k$-domination-covers, or $k$-covers, which are the complements of $k$-dominating graphs. We identify specific properties of $k$-covers, which translate to new properties of domination-critical graphs.

In Section 4.3, we unify the seemingly disparate results that have appeared thus far in our paper: we prove that the same ideals described in Section 4.1 can be represented by a set of polynomials based on $k$-covers, that is, by a set of polynomials based on the complements of domination-critical graphs. By the results in Section 3, we demonstrate that this representation is the universal Gröbner basis of the ideal.

Our paper culminates in Section 5 with an algebraic representation of Vizing's conjecture. This representation is built upon the union of certain varieties and the intersection of certain ideals. We initially present the algebraic version of Vizing's conjecture without respect to a particular representation. We then discuss the consequences of using either of the two representations presented in Sections 4.1 and 4.3. We include comments about computational results and future computational directions, as well as approaches from a purely graph theory perspective. We conclude by clarifying the relationship between universal Gröbner bases and Vizing's conjecture via $k$-covers, and reclaim a known result where Vizing's conjecture holds.

\section{Algebraic Definitions and Background}

In this section, we outline the basic definitions and results concerning ideals and varieties that are utilized throughout the paper, and that are particularly necessary for the algebraic approach to Vizing's conjecture described in Section 5. The results presented in this section are well-known in the field of algebraic geometry and are presented in detail in [11], Chapters 2 and 4. The only new contribution is Lemma 3, which is a small application of well-known results. The ideals referenced throughout are always ideals in polynomial rings, i.e. $I \subseteq \mathbb{K}\left[x_{1}, \ldots, x_{n}\right]$, where $\mathbb{K}$ is an algebraically-closed field. In our case, $\mathbb{K}=\mathbb{C}$.

Given a system of polynomial equations, $f_{1}=f_{2}=\cdots=f_{s}=0$, the ideal associated with the system is $I=\left\langle f_{1}, \ldots, f_{s}\right\rangle$, and the variety associated $I$ (i.e. $V(I)$ ) is the set of common zeros of $\left\{f_{1}, \ldots, f_{s}\right\}$. In other words, $V(I)=\left\{\mathbf{x} \in \mathbb{C}^{n}: f_{1}(\mathbf{x})=\cdots=f_{s}(\mathbf{x})=0\right\}$. An ideal is zero-dimensional if $V(I)$ is finite. Throughout the paper, we will often write a polynomial $f \in I$ as $\sum(\cdot) f_{i}$. In this case, $(\cdot)$ represents the coefficients of the generators $f_{i}$, but because we do not refer to the coefficients explicitly, we do not need to give them individual and precise labels such as $a_{i}$.

Given two ideals, $I=\left\langle f_{1}, \ldots, f_{s}\right\rangle$ and $J=\left\langle g_{1}, \ldots, g_{t}\right\rangle$, the product ideal $I \cdot J$ is the ideal generated by all polynomials $f \cdot g$ with $f \in I$ and $g \in J$. It can be shown ([11], pg 183, Prop. 6) that $I \cdot J$ (often denoted by $I J$ ) is the ideal generated by $\left\langle f_{i} g_{j}: 1 \leqslant i \leqslant\right.$ $s, 1 \leqslant j \leqslant t\rangle$. We summarize the results concerning the varieties of $I$ and $J$ as follows:

$$
V(I) \cup V(J)=V\left(\left\langle f_{i} g_{j}: 1 \leqslant i \leqslant s, 1 \leqslant j \leqslant t\right\rangle\right)=V(I \cdot J)=V(I \cap J) .
$$

An ideal $I$ is radical if $f^{m} \in I$, for some integer $m \geqslant 1$, implies that $f \in I$. Given an ideal $I$, the radical of $I$, denoted $\sqrt{I}$, is the set $\left\{f: f^{m} \in I\right.$ for some integer $\left.m \geqslant 1\right\}$. It 
is easy to see that an ideal $I$ is radical if and only if $I=\sqrt{I}$. We recall the following fact concerning product ideals and radical ideals (left as an exercise in [11]): Given radical ideals $I, J \in \mathbb{K}\left[x_{1}, \ldots, x_{n}\right], \sqrt{I J}=I \cap J$. We note that in one particular case, it is trivial to determine whether or not an ideal is radical.

Lemma 2. ([19], Section 3.7.B, pg. 246) Given a zero-dimensional ideal I, if I contains a univariate square-free polynomial in each variable, then $I$ is radical.

In this case, square-free implies that when a polynomial is decomposed into its unique factorization, there are no repeated factors. For example, $\left(x^{2}+y\right)\left(x^{4}+2 z+3\right)$ is squarefree, but $\left(x^{2}+y\right)\left(x^{4}+2 z+3\right)^{3}$ is not. In particular, Lemma 2 implies that ideals containing $x_{i}^{2}-x_{i}=x_{i}\left(x_{i}-1\right)$ in each variable (i.e., the boolean ideals) are radical. In Section 4 , we will see that all ideals associated with dominating sets (and therefore Vizing's conjecture) are radical for this reason.

In general, it may be quite difficult to determine a basis for $\sqrt{I J}$. However, if $I$ and $J$ are radical ideals via Lemma 2 , and additionally, if the univariate, square-free polynomials in each variable contained in $I, J$ are identical, we can explicitly determine a basis for $\sqrt{I J}$.

Lemma 3. Let $I$ and $J$ be ideals such that $I=\left\langle f_{1}, \ldots f_{s}\right\rangle$ and $J=\left\langle g_{1}, \ldots, g_{t}\right\rangle$. Furthermore, for $1 \leqslant i \leqslant n$, let $f_{i}=g_{i}$ be square-free univariate polynomials in $x_{i}$. Then $\sqrt{I J}=\left\langle f_{i} g_{j}: 1 \leqslant i \leqslant s, 1 \leqslant j \leqslant t\right\rangle+\left\langle f_{i}: 1 \leqslant i \leqslant n\right\rangle$.

Proof. We prove the inclusion in both directions. For convenience, let $M:=\left\langle f_{i} g_{j}: 1 \leqslant\right.$ $i \leqslant s, 1 \leqslant j \leqslant t\rangle+\left\langle f_{i}: 1 \leqslant i \leqslant n\right\rangle$. First, we show that $\sqrt{I J} \subseteq M$. Let $h \in \sqrt{I J}$. Then, there exists an integer $m \geqslant 1$ such that $h^{m} \in I J$. Thus, $h^{m}=\sum(\cdot) f g$. Thus, $h^{m} \in M$. But, by Lemma 2, the ideal $M$ is radical since $M$ contains a square-free univariate polynomial $f_{i}$ in each variable $x_{i}$. Thus, $h^{m} \in M$ implies that $h \in M$.

Conversely, to show that $M \subseteq \sqrt{I J}$, let $h \in M$. Then, $h=\sum(\cdot) f g+\sum(\cdot) f$. Consider

$$
h^{2}=\left(\sum(\cdot) f g+\sum(\cdot) f\right)\left(\sum(\cdot) f g+\sum(\cdot) f\right) .
$$

Any term in the expanded $h^{2}$ can be viewed as $(\cdot) f_{i} g_{j} f_{k} g_{l}=(\cdot) f_{i} g_{j}$, or $(\cdot) f_{i} g_{j} f_{k}=(\cdot) f_{i} g_{j}$, or $(\cdot) f_{i} f_{j}=(\cdot) f_{i} g_{j}$, etc. Thus, any term in the expanded $h^{2}$ can be written as $(\cdot) f g$, with any extra multiplicities in $f$ or $g$ simply folded into the coefficient. Therefore,

$$
h^{2}=\sum(\cdot) f g
$$

and there exists an integer $m=2 \geqslant 1$ such that $h^{m} \in I J$, implying $h \in \sqrt{I J}$.

This brings us to the following critical fact: if $I$ and $J$ are boolean, radical ideals, then by Lemma $3, \sqrt{I J}=\left\langle f_{i} g_{j}: 1 \leqslant i \leqslant s, 1 \leqslant j \leqslant t\right\rangle+\left\langle x_{i}\left(x_{i}-1\right): 1 \leqslant i \leqslant n\right\rangle=I \cap J$.

In Section 4 , when we represent the dominating set problem as a system of polynomial equations, the representations will be boolean, radical ideals as described above. Thus, the basis of their product ideals can be described via Lemma 3. This fact will be vital in Section 5 when we present an algebraic representation of Vizing's conjecture. 


\section{Universal Gröbner Bases and Linear Factors}

In this section, we provide a brief overview of the terminology (from [11], Chap. 2) pertaining to Gröbner bases, and build off the ideas of De Loera in [21] to show that a specific set of linear factor polynomials is a universal Gröbner basis. These "linear factor" ideals allow us to provide a combinatorial interpretation of the universal Gröbner basis of the dominating set ideal defined in Section 4, and will be used in our algebraic exploration of Vizing's conjecture.

A monomial order $\prec$ for the monomials in the polynomial ring $\mathbb{K}\left[x_{1}, \ldots, x_{n}\right]$ is a wellordering which is multiplicative, and for which the constant polynomial is the smallest. The leading term $\operatorname{LT}(f)$ of a polynomial $f \in \mathbb{K}\left[x_{1}, \ldots, x_{n}\right]$ is the largest monomial in $f$ (and its corresponding coefficient) with respect to the monomial order $\prec$. Given an ideal $I$, a finite subset $\mathcal{G}=\left\{g_{1}, \ldots, g_{r}\right\}$ of $I$ is a Gröbner basis of $I$ (with respect to $\prec$ ) if

$$
\left\langle\mathrm{LT}_{\prec}\left(g_{1}\right), \ldots, \mathrm{LT}_{\prec}\left(g_{r}\right)\right\rangle=\left\langle\mathrm{LT}_{\prec}(I)\right\rangle=\left\langle\mathrm{LT}_{\prec}(f): f \in I\right\rangle .
$$

A finite subset $\mathcal{G}$ of $I$ is a universal Gröbner basis of $I$ if it is Gröbner basis of $I$ with respect to any monomial order. Although there are infinitely many monomial orderings, the universal Gröbner basis of $I$ is finite and unique (see [11] for further details). Buchberger's $S$-pair criterion [8] is a specific criterion for determining if a given subset of polynomials is a Gröbner basis of $I$. In order to define Buchberger's S-pair criterion, we fix a monomial order $\prec$ and recall the following notation:

- $\bar{f}^{F}$ denotes the remainder of division of the polynomial $f$ by the ordered $s$-tuple $F=\left(f_{1}, \ldots, f_{s}\right)$ (see [11], Chap. 2, Sec. 3 for details.)

Example: Note that $\overline{\left(x^{2} y+x y^{2}+y^{2}\right)^{\left\{x y-1, y^{2}-1\right\}}}=x+y+1$, since

$$
x^{2} y+x y^{2}+y^{2}=(x+y)(x y-1)+\left(y^{2}-1\right)+x+y+1,
$$

but when the order of $F$ is changed, $\overline{\left(x^{2} y+x y^{2}+y^{2}\right)}\left\{y^{2}-1, x y-1,\right\}=2 x+1$, since

$$
\left(x^{2} y+x y^{2}+y^{2}\right)=(x+1)\left(y^{2}-1\right)+x(x y-1)+2 x+1 .
$$

- $x^{\gamma}$ is the least common multiple of the leading monomial $\operatorname{LM}(f)$ and the leading monomial $\operatorname{LM}(g)$, written as $x^{\gamma}=\operatorname{LCM}(\operatorname{LM}(f), \operatorname{LM}(g))$.

- The $S$-pair of $f$ and $g$ is the combination:

$$
S(f, g)=\frac{x^{\gamma}}{\mathrm{LT}(f)} \cdot f-\frac{x^{\gamma}}{\mathrm{LT}(g)} \cdot g .
$$

We will now characterize Gröbner bases in terms of S-pairs.

Theorem 4 ([8] (also see [11], Chap. 2, Sec. 6 for details)). Given an ideal I, a basis $\mathcal{G}=$ $\left\{g_{1}, \ldots, g_{r}\right\}$ of $I$ is a Gröbner basis for $I$, if and only if, for all pairs $i \neq j, \overline{S\left(g_{i}, g_{j}\right)}=0$. 
We now construct a set of polynomials, where each polynomial is a product of linear factors, and show that if the set satisfies the linear factor criterion, then the set is a universal Gröbner basis. Let $S=\left\{i_{1}, i_{2}, \ldots, i_{k}\right\} \subseteq\{1, \ldots, n\}$, and

$$
P(S):=\left(x_{i_{1}}-1\right)\left(x_{i_{2}}-1\right) \cdots\left(x_{i_{k}}-1\right), \quad \text { and } \quad x(S)=x_{i_{1}} x_{i_{2}} \cdots x_{i_{k}} .
$$

Definition 5. Let $\left\{S_{1}, \ldots, S_{D}\right\}$ be a set such that each $S_{i} \subseteq\{1, \ldots, n\}$ and $\left|S_{i}\right|=d_{i}$. We say that the set $\left\{S_{1}, \ldots, S_{D}\right\}$ satisfies the linear factor criterion if, for any integers $i, j, k$, $S_{k}$ is a not a proper subset of $S_{i} \backslash S_{i} \cap S_{j}$, and $S_{k}$ is a not a proper subset of $S_{j} \backslash S_{i} \cap S_{j}$.

Theorem 6. Let $\left\{S_{1}, \ldots, S_{D}\right\}$ with $S_{i} \subseteq\{1, \ldots, n\}$ be a set that satisfies the linear factor criterion, and let $g_{i}=P\left(S_{i}\right)$. Then $\left\{g_{1}, \ldots, g_{D}\right\}$ is a universal Gröbner basis of $\left\langle g_{1}, \ldots, g_{D}\right\rangle$.

Before we prove this theorem, we present an example.

Example 7. Consider $\mathbb{C}\left[x_{1}, \ldots, x_{12}\right]$, and let $S_{1}=\{1,2,3,5,6\}, S_{2}=\{1,2,3,7,8\}, S_{3}=$ $\{9,10,11,12\}, S_{4}=\{4,8,9\}$. Let $\mathcal{G}=\left\{g_{1}, g_{2}, g_{3}, g_{4}\right\}$ with $g_{i}=P\left(S_{i}\right)$.

$$
\begin{aligned}
& g_{1}:=\left(x_{1}-1\right)\left(x_{2}-1\right)\left(x_{3}-1\right)\left(x_{5}-1\right)\left(x_{6}-1\right), \\
& g_{2}:=\left(x_{1}-1\right)\left(x_{2}-1\right)\left(x_{3}-1\right)\left(x_{7}-1\right)\left(x_{8}-1\right), \\
& g_{3}:=\left(x_{9}-1\right)\left(x_{10}-1\right)\left(x_{11}-1\right)\left(x_{12}-1\right), \\
& g_{4}:=\left(x_{4}-1\right)\left(x_{8}-1\right)\left(x_{9}-1\right) .
\end{aligned}
$$

Since $S_{1} \cap S_{2}=\{1,2,3\}, S_{1} \backslash\left(S_{1} \cap S_{2}\right)=\{5,6\}$, and $S_{2} \backslash\left(S_{1} \cap S_{2}\right)=\{7,8\}$. Neither $S_{3}$ nor $S_{4}$ is a proper subset of $S_{1} \backslash\left(S_{1} \cap S_{2}\right)$ or $S_{2} \backslash\left(S_{1} \cap S_{2}\right)$. This is true for all $i, j$ pairs, thus $\left\{S_{1}, S_{2}, S_{3}, S_{4}\right\}$ satisfies the linear factor criterion. Additionally, note that $\operatorname{LT}\left(g_{i}\right)$ is always $x\left(S_{i}\right)$, regardless of the specified monomial order. Thus, we see

$$
\begin{aligned}
S\left(g_{2}, g_{4}\right)= & \frac{x_{1} x_{2} x_{3} x_{4} x_{7} x_{8} x_{9}}{x_{1} x_{2} x_{3} x_{7} x_{8}} g_{2}-\frac{x_{1} x_{2} x_{3} x_{4} x_{7} x_{8} x_{9}}{x_{4} x_{8} x_{9}} g_{4}=x_{4} x_{9} g_{2}-x_{1} x_{2} x_{3} x_{7} g_{4} \\
= & \left(x_{4}+x_{9}-1\right) g_{2}-\left(x_{1} x_{2} x_{3}+x_{1} x_{2} x_{7}+x_{1} x_{3} x_{7}+x_{2} x_{3} x_{7}-x_{1} x_{2}-x_{1} x_{3}\right. \\
& \left.-x_{1} x_{7}-x_{2} x_{3}-x_{2} x_{7}-x_{3} x_{7}+x_{1}+x_{2}+x_{3}+x_{7}-1\right) g_{4} .
\end{aligned}
$$

Regardless of the monomial order, the leading terms of the coefficients of $g_{2}$ and $g_{4}$ are not divisible by the leading terms of $g_{1}, g_{2}, g_{3}$ or $g_{4}$, Thus, $\overline{S\left(g_{2}, g_{4}\right)}=0$. Performing similar calculations for all other pairs can quickly show that $\left\{g_{1}, \ldots, g_{4}\right\}$ is the universal Gröbner basis of $\left\langle g_{1}, \ldots, g_{4}\right\rangle$.

For simplicity of notation, let $S_{\text {only }}^{i}=S_{i} \backslash\left(S_{i} \cap S_{j}\right)$ and $S_{\text {only }}^{j}=S_{j} \backslash\left(S_{i} \cap S_{j}\right)$. Furthermore, let $S_{\subseteq}^{i j}$ be a subset of $S_{i} \cap S_{j}, S_{\subseteq \text { only }}^{i}$ be a subset of $S_{\text {only }}^{i}$, and $S_{\subseteq \text { only }}^{j}$ be a subset of $S_{\text {only }}^{j}$. Note that

$$
\left|S_{\text {only }}^{j}\right|+d_{i}=\left|S_{\text {only }}^{i}\right|+d_{j}
$$


Proof. We will show that the set of generators $\mathcal{G}=\left\{g_{1}, \ldots, g_{D}\right\}$ satisfies Buchberger's S-pair criterion. Consider any two polynomials $g_{i}, g_{j}$. We must show $\overline{S\left(g_{i}, g_{j}\right)^{\mathcal{G}}}=0$. We claim

$$
\begin{aligned}
S\left(g_{i}, g_{j}\right) & =\frac{x\left(S_{i} \cup S_{j}\right)}{x\left(S_{i}\right)} g_{i}-\frac{x\left(S_{i} \cup S_{j}\right)}{x\left(S_{j}\right)} g_{j}=x\left(S_{\text {only }}^{j}\right) g_{i}-x\left(S_{\text {only }}^{i}\right) g_{j} \\
& =\left(\operatorname{LT}\left(P\left(S_{\text {only }}^{j}\right)\right)-P\left(S_{\text {only }}^{j}\right)\right) g_{i}-\left(\operatorname{LT}\left(P\left(S_{\text {only }}^{i}\right)\right)-P\left(S_{\text {only }}^{i}\right)\right) g_{j} .
\end{aligned}
$$

Before we prove the equality between lines 2 and 3, we note that, if true, we have already shown that $\overline{S\left(g_{i}, g_{j}\right)}=0$. This can be seen by noting that, since $\left\{S_{1}, \ldots, S_{D}\right\}$ satisfies the linear factor criterion, given integers $i, j, k, S_{k}$ is not a proper subset of $S_{\text {only }}^{i}$ and $S_{k}$ is not a proper subset of $S_{\text {only }}^{j}$. Additionally, regardless of the monomial order, the leading term of $g_{i}$ is $x\left(S_{i}\right)$, and the leading term of $\left(\operatorname{LT}\left(P\left(S_{\text {only }}^{j}\right)\right)-P\left(S_{\text {only }}^{j}\right)\right)$ is a proper subset of $S_{\text {only }}^{j}$ (similarly, $\left(\operatorname{LT}\left(P\left(S_{\text {only }}^{i}\right)\right)-P\left(S_{\text {only }}^{i}\right)\right)$ is a proper subset of $\left.S_{\text {only }}^{i}\right)$. Thus, $\operatorname{LT}\left(g_{k}\right)$ does not divide either $\left(\operatorname{LT}\left(P\left(S_{\text {only }}^{j}\right)\right)-P\left(S_{\text {only }}^{j}\right)\right)$ or $\left(\operatorname{LT}\left(P\left(S_{\text {only }}^{i}\right)\right)-P\left(S_{\text {only }}^{i}\right)\right)$, and we can see that $\overline{S\left(g_{i}, g_{j}\right)} \mathcal{G}=0$.

In order to prove the equality between lines 2 and 3 , we must show that every monomial in line 2 either cancels within line 2, or appears in line 3 with the same coefficient, and vice versa.

Let $x(M)$ be a monomial appearing in line 2. Either

$$
M=S_{\text {only }}^{j} \cup S_{\subseteq}^{i j} \cup S_{\subseteq \text { only }}^{i}, \quad \text { or } \quad M=S_{\text {only }}^{i} \cup S_{\subseteq}^{i j} \cup S_{\subseteq \text { only }}^{j} .
$$

We note that $x(M)$ appears in both $x\left(S_{\text {only }}^{j}\right) g_{i}$ and $x\left(S_{\text {only }}^{i}\right) g_{j}$ only when $S_{\subseteq \text { only }}^{i}=S_{\text {only }}^{i}$ and $S_{\subseteq \text { only }}^{j}=S_{\text {only }}^{j}$. In this case, the coefficient for $x(M)$ is $(-1)^{C}-(-1)^{D}$ where

$$
C=d_{i}-\left(\left|S_{\subseteq}^{i j}\right|+\left|S_{\text {only }}^{i}\right|\right), \quad \text { and } \quad D=d_{j}-\left(\left|S_{\subseteq}^{i j}\right|+\left|S_{\text {only }}^{j}\right|\right) .
$$

This formula can be explained by noting that, in any polynomial that is the product of linear factors, the coefficient for a given monomial is $(-1)^{C}$ where $C$ is the degree of the polynomial minus the degree of the given monomial. For example, consider $f:=$ $\left(x_{1}-1\right)\left(x_{2}-1\right)\left(x_{3}-1\right)\left(x_{7}-1\right)$. The coefficient for the monomial $x_{1} x_{2} x_{3}$ is $(-1)^{4-3}=-1$, and the coefficient for $x_{2} x_{7}$ is $(-1)^{4-2}=1$.

Using the identity given in Eq. 1 , we note that $d_{i}-\left|S_{\text {only }}^{i}\right|=d_{j}-\left|S_{\text {only }}^{j}\right|$. Thus, $C=D$, and the monomial cancels.

We will now consider the case where $M=S_{\text {only }}^{j} \cup S_{\subseteq}^{i j} \cup S_{\subseteq \text { only }}^{i}$ and $S_{\subseteq \text { only }}^{i} \neq S_{\text {only }}^{i}$. In this case, $x(M)$ appears in line 2 in the product $x\left(S_{\text {only }}^{j}\right) g_{i}$, and the coefficient for $x(M)$ is $(-1)^{C}$ where

$$
C=d_{i}-\left(\left|S_{\subseteq}^{i j}\right|+\left|S_{\subseteq \text { only }}^{i}\right|\right) .
$$


In line $3, x(M)$ appears only in the product $-\left(\operatorname{LT}\left(P\left(S_{\text {only }}^{i}\right)\right)-P\left(S_{\text {only }}^{i}\right)\right) g_{j}$, and the coefficient for $x(M)$ is $(-1)^{C^{\prime}}$ where

$$
C^{\prime}=\left|S_{\text {only }}^{i}\right|-\left|S_{\subseteq \text { only }}^{i}\right|+d_{j}-\left(\left|S_{\subseteq}^{i j}\right|+\left|S_{\text {only }}^{j}\right|\right)+2 .
$$

But using the identity from Eq. 1, and substituting for $d_{j}$, we see

$$
\begin{aligned}
C^{\prime} & =\left|S_{\text {only }}^{i}\right|-\left|S_{\subseteq \text { only }}^{i}\right|+\left(\left|S_{\text {only }}^{j}\right|+d_{i}-\left|S_{\text {only }}^{i}\right|\right)-\left(\left|S_{\subseteq}^{i j}\right|+\left|S_{\text {only }}^{j}\right|\right)+2 \\
& =d_{i}-\left|S_{\subseteq \text { only }}^{i}\right|-\left|S_{\subseteq}^{i j}\right|+2 .
\end{aligned}
$$

Thus, $C$ is the same parity as $C^{\prime}$, which implies that the coefficient for $x(M)$ in line 2 , and the coefficient for $x(M)$ in line 3 are the same.

The case where $M=S_{\text {only }}^{i} \cup S_{\subseteq}^{i j} \cup S_{\subseteq \text { only }}^{j}$ and $S_{\subseteq \text { only }}^{j} \neq S_{\text {only }}^{j}$ is the same. Thus, we have shown that every monomial in line 2 either cancels, or appears in line 3 with the same coefficient.

We must now show that every monomial in line 3 either cancels, or appears in line 2 with the same coefficient. Let $x(M)$ be a monomial appearing in line 3. Either

$$
M=S_{\subsetneq \text { only }}^{j} \cup S_{\subseteq}^{i j} \cup S_{\subseteq \text { only }}^{i}, \quad \text { or } \quad M=S_{\subsetneq \text { only }}^{i} \cup S_{\subseteq}^{i j} \cup S_{\subseteq \text { only }}^{j} .
$$

We note that in each case, $M$ contains a proper subset of $S_{\text {only }}^{j}$ or a proper subset of $S_{\text {only }}^{i}$. Additionally, the two cases when $S_{\subseteq \text { only }}^{i}=S_{\text {only }}^{i}$, or $S_{\subseteq \text { only }}^{j}=S_{\text {only }}^{j}$ have already been explicated above. Thus, in both cases, we have already shown that $x(M)$ appears in line 2 with the same coefficient.

We will now consider monomials of the form $x(M)$ where $M=S_{\subsetneq \text { only }}^{j} \cup S_{\subset}^{i j} \cup S_{\subsetneq \text { only }}^{i}$, and show that these monomials cancel within line 3 . The coefficient for $x(M)$ is $(-1)^{C}-(-1)^{D}$ where

$$
\begin{aligned}
& C=\left|S_{\text {only }}^{j}\right|-\left|S_{\subsetneq \text { only }}^{j}\right|+d_{i}-\left(\left|S_{\subseteq}^{i j}\right|+\left|S_{\subsetneq \text { only }}^{i}\right|\right), \quad \text { and } \\
& D=\left|S_{\text {only }}^{i}\right|-\left|S_{\subsetneq \text { only }}^{i}\right|+d_{j}-\left(\left|S_{\subseteq}^{i j}\right|+\left|S_{\subsetneq \text { only }}^{i}\right|\right)+2 .
\end{aligned}
$$

As before, using the identify from Eq. 1 and substituting for $d_{j}$, we see $C$ is the same parity as $D$. Thus, $(-1)^{C}-(-1)^{D}=0$, and the monomial cancels.

Thus, we have shown that every monomial in line 2 either cancels within line 2, or appears in line 3 with the same coefficient, and vice versa. Additionally, we have not utilized the properties of any particular monomial order to do so. Thus, we have shown that $\left\{g_{1}, \ldots, g_{D}\right\}$ is a universal Gröbner basis for $\left\langle g_{1}, \ldots, g_{D}\right\rangle$.

The following corollary extends the theorem above to include "boolean" polynomials of the form $x_{i}^{2}-x_{i}$. This is particularly interesting because boolean polynomials are a common ingredient in non-linear models of combinatorial problems.

Corollary 8. Let $\left\{S_{1}, \ldots, S_{D}\right\}$ with $S_{i} \subseteq\{1, \ldots, n\}$ (and $\left|S_{i}\right|>1$ ) be a set that satisfies the linear factor criterion. Let $g_{i}=P\left(S_{i}\right)$, and let $b_{i}=x_{i}^{2}-x_{i}$. Then $\mathcal{G}=\left\{b_{1}, \ldots, b_{n}\right\} \cup$ $\left\{g_{1}, \ldots, g_{D}\right\}$ is the universal Gröbner basis for $\left\langle b_{1}, \ldots, b_{n}\right\rangle+\left\langle g_{1}, \ldots, g_{D}\right\rangle$. 
We observe that $x_{i}-1$ and $x_{i}^{2}-x_{i}$ are redundant equations, which explains the extra condition $\left|S_{i}\right|>1, \forall i$.

Proof. By Theorem 6, we have already seen that $\left\{g_{1}, \ldots, g_{D}\right\}$ is a universal Gröbner basis. Therefore, in order to show that $\mathcal{G}$ satisfies Buchberger's criterion, it remains to show that $\overline{S\left(b_{i}, b_{j}\right)}{ }^{\mathcal{G}}=\overline{S\left(b_{i}, g_{j}\right)}{ }^{\mathcal{G}}=0$, without relying on properties of a particular monomial order, .

Because monomial orders are by definition well-orders, $\operatorname{LT}\left(b_{i}\right)$ is always $x_{i}^{2}$, and $\operatorname{LT}\left(g_{i}\right)$ is always $x\left(S_{i}\right)$, regardless of the specific monomial order.

We will first show that $\overline{S\left(b_{i}, b_{j}\right)}$ G $=0$ for $i \neq j$. Note that

$$
S\left(b_{i}, b_{j}\right)=\frac{x_{i}^{2} x_{j}^{2}}{x_{i}^{2}}\left(x_{i}^{2}-x_{i}\right)-\frac{x_{i}^{2} x_{j}^{2}}{x_{j}^{2}}\left(x_{j}^{2}-x_{j}\right)=x_{i}^{2} x_{j}-x_{i} x_{j}^{2}=x_{j}\left(x_{i}^{2}-x_{i}\right)-x_{i}\left(x_{j}^{2}-x_{j}\right) .
$$

Since $\left|S_{i}\right|>1$, the coefficients $-x_{i}$ and $x_{j}$ are not divisible by $\operatorname{LT}\left(g_{k}\right), \forall k$. Thus, $\overline{S\left(b_{i}, b_{j}\right)} \mathcal{G}=0$.

We will now show that $\overline{S\left(b_{i}, g_{j}\right)} \mathcal{G}=0$, for all $i, j$ pairs.

- Case 1: Choose an $i$ such that $i \notin S_{j}$, and write $g_{j}:=x\left(S_{j}\right)+P_{\text {rest. }}$. Note

$$
\begin{aligned}
S\left(b_{i}, g_{j}\right) & =\frac{x_{i}^{2} x\left(S_{j}\right)}{x_{i}^{2}}\left(x_{i}^{2}-x_{i}\right)-\frac{x_{i}^{2} x\left(S_{j}\right)}{x\left(S_{j}\right)}\left(x\left(S_{j}\right)+P_{\text {rest }}\right)=-x_{i} x\left(S_{j}\right)-x_{i}^{2}\left(P_{\text {rest }}\right) \\
& =-x_{i}\left(x\left(S_{j}\right)+P_{\text {rest }}\right)-P_{\text {rest }}\left(x_{i}^{2}-x_{i}\right)=-x_{i} g_{j}-P_{\text {rest }}\left(x_{i}^{2}-x_{i}\right) .
\end{aligned}
$$

By the linear factor criterion, $\operatorname{LT}\left(P_{\text {rest }}\right)$ is not divisible by $\operatorname{LT}\left(g_{k}\right)$ for any $k$. Thus, $\overline{S\left(b_{i}, g_{j}\right)^{\mathcal{G}}}=0$.

- Case 2: Choose an $i$ such that $i \in S_{j}$, and write $g_{j}:=x\left(S_{j}\right)-x\left(S_{j} \backslash i\right)+P_{\text {rest }}$. Since $i \in S_{j}$, this implies that $\operatorname{LCM}\left(\operatorname{LT}\left(b_{i}\right), \operatorname{LT}\left(g_{j}\right)\right)=x_{i} x\left(S_{j}\right)=x_{i}^{2} x\left(S_{j} \backslash i\right)$. Then

$$
\begin{aligned}
S\left(b_{i}, g_{j}\right) & =\frac{x_{i}^{2} x\left(S_{j} \backslash i\right)}{x_{i}^{2}}\left(x_{i}^{2}-x_{i}\right)-\frac{x_{i} x\left(S_{j}\right)}{x\left(S_{j}\right)}\left(x\left(S_{j}\right)-x\left(S_{j} \backslash i\right)+P_{\text {rest }}\right)=-x_{i} P_{\text {rest }} \\
& =\left(x\left(S_{j} \backslash i\right)-P\left(S_{j} \backslash i\right)\right)\left(x_{i}^{2}-x_{i}\right),
\end{aligned}
$$

and $\overline{S\left(b_{i}, g_{j}\right)}{ }^{\mathcal{G}}=0$ by the linear factor criterion. The equality of lines 4 and 5 can be seen as follows. The polynomial $x\left(S_{j} \backslash i\right)-P\left(S_{j} \backslash i\right)$ is the polynomial $P\left(S_{j} \backslash i\right)$ with the signs changed and the leading term removed. When multiplied by a positive $x_{i}^{2}$ and a negative $x_{i}$, every monomial appearing in the product also appears in $-x_{i}\left(P_{\text {rest }}\right)$. To see this clearly, consider a monomial $x(M)$ where $M$ is a proper subset of $S_{j}$ (this is the only kind of monomial appearing in $P_{\text {rest }}$ on line 4). There are two cases:

- Case 2a: $i \in M$. When $x(M)$ is multiplied by $-x_{i}$ (line 4), the sign of the leading coefficient changes, and the degree increases by one. In other 
words, $x_{i} x(M)$ appears in expanded product $-x_{i}\left(P_{\text {rest }}\right)$ with leading coefficient $(-1) \cdot(-1)^{d_{j}-|M|}$. However, $x(M \backslash i)$ appears in $P\left(S_{j} \backslash i\right)$ with the same parity sign as $x(M)$ in $P_{\text {rest }}\left(\right.$ i.e., $\left.(-1)^{\left(d_{j}-1\right)-(|M|-1)}\right)$. Thus, the product $-P\left(S_{j} \backslash i\right)\left(x_{i}^{2}\right)$ produces the same monomial with the same leading coefficient as $-x_{i}\left(P_{\text {rest }}\right)$ (line 5) and equality between lines 4 and 5 is preserved.

- Case 2b: $i \notin M$. In this case, $x(M)$ appears in $P\left(S_{j} \backslash i\right)$, but with opposite sign as $x(M)$ in $P_{\text {rest }}$. In particular, $x(M)$ in $P_{\text {rest }}$ has coefficient $(-1)^{d_{j}-|M|}$ as before, but $x(M)$ in $P\left(S_{j} \backslash i\right)$ has coefficient $(-1)^{\left(d_{j}-1\right)-|M|}$. Thus, the product $-P\left(S_{j} \backslash i\right)\left(-x_{i}\right)$ produces the same monomial with the same leading coefficient as $-x_{i}\left(P_{\text {rest }}\right)$ and equality between lines 4 and 5 is preserved

In cases $2 \mathrm{a}$ and $2 \mathrm{~b}$, we demonstrated that the monomials produced by $-P\left(S_{j} \backslash i\right)\left(x_{i}^{2}\right)$ and $-P\left(S_{j} \backslash i\right)\left(-x_{i}\right)$ respectively, appear in line 4 . Thus, we have accounted for every monomial in line 5 , and we have shown that line 4 is equal to line 5 .

We have shown that $\overline{S\left(b_{i}, b_{j}\right)}=0$ for $i \neq j$, and that $\overline{S\left(b_{i}, g_{j}\right)}=0$, for all $i, j$ pairs, without relying on any properties of a monomial order. Since we have already shown that $\left\{g_{1}, \ldots, g_{D}\right\}$ is a universal Gröbner basis, this means that we have shown that $\left\{b_{1}, \ldots, b_{n}\right\} \cup\left\{g_{1}, \ldots, g_{D}\right\}$ is a universal Gröbner basis of $\left\langle b_{1}, \ldots, b_{n}\right\rangle+\left\langle g_{1}, \ldots, g_{D}\right\rangle$. This concludes our proof.

In Section 4.3, we will represent the dominating set problem as a system of polynomial equations in such a way that the representation is already a universal basis. This representation follows the work of De Loera [21], where the graph coloring problem is also represented in such a way that it is a universal Gröbner basis. These kinds of representations may prove useful in the context of combinatorial ideal membership questions, and also with the advance of algorithms specifically tailored for finding universal Gröbner basis, such as [4].

\section{Dominating Sets, Ideals and Gröbner Bases}

In this section, we begin by formulating the dominating set problem as a system of polynomial equations. We extend this formulation to include a dominating set in a graph $G$, a dominating set in a graph $H$, and a dominating set in the product graph $G \square H$. We refer to this formulation (linking graphs $G, H$, and $G \square H$ ) in Section 5 during the algebraic formulation of Vizing's conjecture. In Section 4.2, we introduce the idea of a $k$ domination cover or a $k$-cover. We explain the relation between $k$-covers and dominationcritical graphs, and provide various examples of $k$-covers. We also prove several properties of $k$-covers, and provide a conjecture for future work. We unify these ideas in Section 4.3 by showing that $k$-covers provide the combinatorial interpretation of the universal Gröbner basis of the ideals described in Section 4.1. Thus, the purpose of this section is to explore two different non-linear models of the dominating set problem based on two different combinatorial properties, and surprisingly, the second representation is the universal Gröbner basis of the first. 


\subsection{Dominating Sets and Systems of Polynomial Equations}

Let $S_{k}^{n}$ represent the set of $k$-subsets of $\{1,2, \ldots, n\}$. Thus, $\left|S_{k}^{n}\right|=\left(\begin{array}{l}n \\ k\end{array}\right)$, and $S \in S_{k}^{n}$ implies that $S \subseteq\{1,2, \ldots, n\}$ is a particular subset with $|S|=k$. In the following system of polynomial equations, there is one binary decision variable for every possible edge in a graph with $n$ vertices. Thus, the variable $e_{i j}$ is 1 if the edge between vertex $i$ and vertex $j$ exists in the graph, and 0 otherwise. Since our graphs are undirected, we implicitly assume the substitution $e_{j i}=e_{i j}$ whenever $j>i$.

Theorem 9. There is a bijection between the set of solutions of the following system of equations and the set of labeled graphs $G$ in $n$ vertices with a dominating set of size $k$.

$$
\begin{aligned}
e_{i j}^{2}-e_{i j} & =0, \quad \text { for } 1 \leqslant i<j \leqslant n, \\
\prod_{S \in S_{k}^{n}}\left(\sum_{i \notin S}\left(\prod_{j \in S}\left(e_{i j}-1\right)\right)\right) & =0 .
\end{aligned}
$$

Example 10. Let $n=3$ and $k=1$. The variables in the system of polynomial equations defined by Theorem 9 are $e_{12}, e_{13}$ and $e_{23}$. Furthermore, $S_{1}^{3}=\{\{1\},\{2\},\{3\}\}$.

For the second equation, we have:

$$
\left(\left(e_{21}-1\right)+\left(e_{31}-1\right)\right)\left(\left(e_{12}-1\right)+\left(e_{32}-1\right)\right)\left(\left(e_{13}-1\right)+\left(e_{23}-1\right)\right)=0 .
$$

However, as noted above, since the graph is undirected, we implicitly assume the substitution $e_{21}=e_{12}, e_{31}=e_{13}$ and $e_{32}=e_{23}$. Thus, the system of equations is as follows:

$$
\begin{aligned}
e_{12}^{2}-e_{12}=0, \quad e_{13}^{2}-e_{13}=0, \quad e_{23}^{2}-e_{23} & =0 \\
\left(\left(e_{12}-1\right)+\left(e_{13}-1\right)\right)\left(\left(e_{12}-1\right)+\left(e_{23}-1\right)\right)\left(\left(e_{13}-1\right)+\left(e_{23}-1\right)\right) & =0 .
\end{aligned}
$$

The solutions to the system of equations are in bijection with the labeled graphs on three vertices with a dominating set of size one:
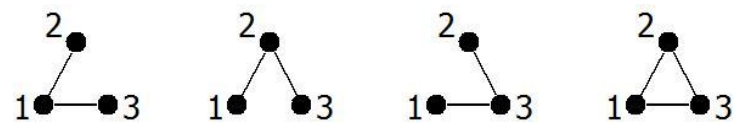

Proof. We refer to the system of polynomial equations defined by Theorem 9 as $\star$ throughout this proof. We will define a map $\phi$ between the solutions of $\star$ and the labeled graphs $G$ in $n$ vertices with a dominating set of size $k$, and show that $\phi$ is a bijection between the two sets.

To deal with the problem of graph isomorphisms, we consider a set of $n$ vertices with a fixed labeling 1 through $n$. Thus, two graphs $G$ and $G^{\prime}$ on this labeled set of $n$ vertices are equal if and only if $(i, j) \in E(G)$ implies that $(i, j) \in E\left(G^{\prime}\right)$. In other words, if $G$ and $G^{\prime}$ are isomorphic, they are not necessarily equal under this definition. Then, our map $\phi$ simply takes a solution to $\star$, and converts it to a graph $G$ on the labeled set of $n$ 
vertices by adding the edges $(i, j)$ to $E(G)$ if and only if the variable $e_{i j}=1$. Then, $\phi$ is one-to-one, since given any two solutions $s$ and $s^{\prime}$, if $G=\phi(s)=\phi\left(s^{\prime}\right)=G^{\prime}$, then clearly $s=s^{\prime}$.

We will now show that the image of $\phi$ is a subset of the set of labeled graphs $G$ with a dominating set of size $k$. The boolean equations $e_{i j}^{2}-e_{i j}=0$ force every variable $e_{i j}$ to be zero or one; thus, the boolean equations turn edges "on" or "off" when applying the map $\phi$, and every solution corresponds to a particular graph $G$. We must now show that since the solution satisfies Eq. 6, the particular graph $G$ formed from the solution has a dominating set of size $k$. Let $A$ and $B$ denote different pieces of Eq. 6 as follows.

$$
\prod_{S \in S_{k}^{n}} \underbrace{(\sum_{i \notin S} \underbrace{\left(\prod_{j \in S}\left(e_{i j}-1\right)\right)}_{A})}_{B}=0 .
$$

The equation is only satisfied if one of the inner summations $B$ (corresponding to some set $S$ ) is zero. The value of an individual summand $A$ in $B$ is either zero or \pm 1 . However, two different summands $A, A^{\prime}$ in the summation $B$ can never mutually cancel, since if they are both non-zero, they are both $-1^{|S|}$. Thus, they are either both -1 or both +1 . Therefore, a summation $B$ is only zero if every individual summand $A$ is also zero, and $A$ is only zero if at least one edge variable has value one. Therefore, every $i \notin S$ is adjacent to a $j \in S$. In other words, $G$ has a dominating set of size $k$.

Having shown that the image of $\phi$ is a subset of the set of labeled graphs $G$ with a dominating set of size $k$, we must now show that $\phi$ is onto, or that given any graph $G$ with a dominating set of size $k, \phi^{-1}(G)$ is a solution to $\star$. The map $\phi^{-1}(G)$ would be applied as follows: if the edge $e_{i j}$ is present in $G$, turn it "on" by setting the variable $e_{i j}=1$. If the edge $e_{i j}$ is not present in $G$, turn the variable $e_{i j}$ "off" by setting the variable $e_{i j}=0$. Clearly, the boolean equations $e_{i j}^{2}-e_{i j}=0$ are satisfied. Since the graph $G$ has a dominating set of size $k$, let $S=\left\{i_{1}, \ldots, i_{k}\right\}$ be such a dominating set. Thus, every vertex $i$ not in the dominating set $S$ must be adjacent to a vertex $j$ that is in the dominating set $S$. In other words, an edge $e_{i j}$ is "on" from $i \notin S$ to $j \in S$, and the $\left(e_{i j}-1\right)$ term in the product corresponding to the dominating set $S$ is equal to zero. Since every vertex $i \notin S$ must satisfy this condition, this implies that every summand in the summation (corresponding to the dominating set $S$ )

$$
\sum_{i \notin S}\left(\prod_{j \in S}\left(e_{i j}-1\right)\right)
$$

is equal to zero, and thus the entire summation is equal to zero. Since Eq. 6 is a product of summations, the equation is satisfied.

Thus, $\phi$ is one-to-one and onto, and $\phi$ is a bijection between solutions of $\star$ and the set of labeled graphs $G$ with a dominating set of size $k$.

We will now use the system of polynomial equations defined in Theorem 9 as a building block to model dominating sets in $G, H$ and $G \square H$. 
Theorem 11. There is a bijection between the set of solutions of the following system of polynomial equations and the set of labeled graphs $G, H$ in $n, n^{\prime}$ vertices with dominating sets of size $k, l$ respectively such that their Cartesian product graph $G \square H$ has a dominating set of size $r$.

Representing a graph $G$ in $n$ vertices with a dominating set of size $k$ :

$$
\begin{aligned}
& e_{i j}^{2}-e_{i j}=0, \quad \text { for } 1 \leqslant i<j \leqslant n, \\
& \underbrace{\prod_{S \in S_{k}^{n}}\left(\sum_{i \notin S}\left(\prod_{j \in S}\left(e_{i j}-1\right)\right)\right)}_{P_{G}^{K}}=0 .
\end{aligned}
$$

Representing the graph $H$ in $n^{\prime}$ vertices with a dominating set of size l:

$$
\begin{aligned}
& e_{i j}^{\prime 2}-e_{i j}^{\prime}=0, \text { for } 1 \leqslant i<j \leqslant n^{\prime}, \\
& \underbrace{\prod_{S \in S_{l}^{n^{\prime}}}\left(\sum_{i \notin S}\left(\prod_{j \in S}\left(e^{\prime}{ }_{i j}-1\right)\right)\right)}_{P_{H}^{l}}=0 .
\end{aligned}
$$

Representing the Cartesian product graph $G \square H$ with a dominating set of size $r$ :

$$
\underbrace{\prod_{S \in S_{r}^{n n^{\prime}}}\left(\sum_{g h \notin S}\left(\prod_{g^{\prime} h \in S}\left(e_{g g^{\prime}}-1\right) \prod_{g h^{\prime} \in S}\left(e^{\prime}{ }_{h h^{\prime}}-1\right)\right)\right)}_{P_{G \square H}^{r}}=0 . \quad \quad\left(P_{G \square H}^{r}\right)
$$

Proof. It is clear from Theorem 9 that there is a bijection between the solutions of the equations representing graphs $G$ and $H$ and the set of graphs in $n, n^{\prime}$ vertices with dominating sets of size $k, l$ respectively. The equation representing $G \square H$ is of the same form, except that this equation takes into account the unique structure of the product graph. For example, if a vertex not in $S$ is adjacent to a vertex in $S$, the adjacency is either due to an edge in $G$, or an edge in $H$. In particular, $g h \notin S$ is either adjacent to a vertex $g^{\prime} h \in S$ if the edge $\left(g, g^{\prime}\right)$ is "on" in $G$, or $g h \notin S$ is adjacent to a vertex $g h^{\prime} \in S$ if the edge $\left(h, h^{\prime}\right)$ is "on" in $H$. Thus, the proof of the bijection follows the logic of the proof of Theorem 9.

Since the system of polynomial equations described in Theorem 11 depends on the variables $n, k, n^{\prime}, l$ and $r$, we define the ideal $I\left(n, k, n^{\prime}, l, r\right)$ as

$$
I\left(n, k, n^{\prime}, l, r\right):=\left\langle P_{G}^{k}, P_{H}^{l}, P_{G \square H}^{r}, e^{2}-e\right\rangle,
$$

where $e^{2}-e$ denotes the entire set of boolean edge equations

$$
\left\{e_{i j}^{2}-e_{i j}: 1 \leqslant i<j \leqslant n, e^{\prime 2}{ }_{i j}-e_{i j}^{\prime}: 1 \leqslant i<j \leqslant n^{\prime},\right\} .
$$

THe ELECTROnic Journal of COMBinatorics 19(2) (2012), \#P1 
We note that $I\left(n, k, n^{\prime}, l, r\right)$ is radical by Lemma 2 , since the boolean equations $e^{2}-e$ are univariate and square-free. We now define $k$-covers, which provide a combinatorial interpretation of the universal Gröbner basis of $I\left(n, k, n^{\prime}, l, r\right)$ in Section 4.3. We note that what is important about the next section is not the uniqueness or complexity of the definition of $k$-covers, but rather the interesting relationship between the ideal $I\left(n, k, n^{\prime}, l, r\right)$, the combinatorial idea of a $k$-cover, and the universal Gröbner basis of the ideal.

\section{$4.2 k$-Covers and $k$-Dominating Sets}

We begin by recalling that a graph $G$ is domination-critical if, for any two non-adjacent vertices $u, v$, the graph $G^{\prime}:=G+(u, v)$ has $\gamma\left(G^{\prime}\right)=\gamma(G)-1$. In other words, a graph $G$ is domination-critical if, whenever any edge is added to $G$, the domination number decreases by one. Thus, $k$-domination-critical graphs are created from $k$-dominating graphs by adding edges. In this section, we study the complements of domination-critical graphs. We define a $k$-domination-cover (or $k$-cover) as analogous to a $k$-dominating graph, and a minimal $k$-cover as analogous to a $k$-domination-critical graph. Just as a dominationcritical graph is obtained from a $k$-dominating graph by adding edges, a minimal $k$-cover is obtained from a $k$-cover by removing edges.

Before we begin, we recall a few definitions. Let $K_{n}$ denote the complete graph in $n$ vertices (an $n$-clique). For $S \subseteq V(G)$, the open neighborhood of $S$ (denoted by $N_{G}(S)$, or simply $N(S)$ when the context of the graph is clear) is defined to be

$$
N_{G}(S):=\{v:(u, v) \in E(G) \text { and } u \in S \text { and } v \notin S\} \text {. }
$$

The common neighborhood of $S \subseteq V(G)$ (denoted by $c m N_{G}(S)$, or simply $c m N(S)$ when the context of the graph is clear) is defined to be

$$
c m N_{G}(S):=\bigcap_{u \in S} N_{G}(\{u\})
$$

Example 12. In the following example, let $S=\{0,1,3\}$. Then $\operatorname{cm} N(S)=\{6\}$.

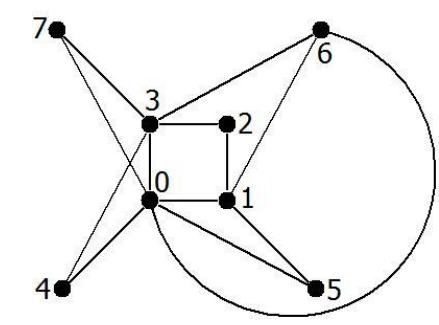

Definition 13. A graph $C$ is a $k$-cover if $\operatorname{cmN}_{C}(S) \neq \emptyset$ for all $S \subseteq V(C)$ with $|S|=k-1$.

We say that a graph $C$ is a $k$-cover if every set of size $k-1$ has a common neighbor. We note that $k$-covers are only defined for $k \geqslant 2$.

Definition 14. A $k$-cover $C$ is minimal if for all $e \in E(C), C^{\prime}:=C-e$ is not a $k$-cover. 
Example 15. Here we see a minimal 3-cover (left) and its complement (right).
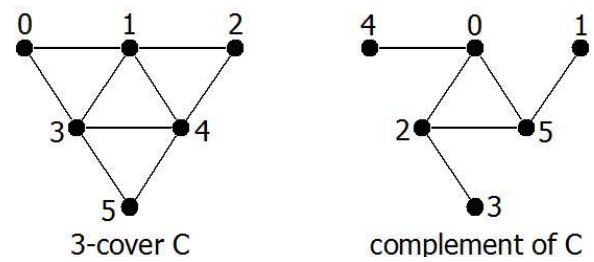

We refer to graphs of the type described by Definition 13 as $k$-covers, because we are taking $n$ vertices and then "covering" those $n$ vertices with $k$-cliques such that the $k$-cliques intersect in a very particular way. Notice that the 3-cover illustrated in Ex. 15 takes six vertices, and then "covers" those six vertices with triangles such that any subset of size two has a common neighbor.

Proposition 16. Given a $k$-cover $C$, every $v \in V(C)$ appears in a clique of size $k$.

Proof. Since every set of size $k-1$ in $V(C)$ has a common neighbor, every vertex has at least one outgoing edge. Thus, for any $v_{1} \in V(C), v_{1}$ is adjacent to some $v_{2}$. If $2 \leqslant k-1$, both $v_{1}$ and $v_{2}$ are each adjacent to a third vertex $v_{3}$. In other words, $v_{1}, v_{2}$ and $v_{3}$ form a 3 -clique. By repeating this process $k-1$ times, we form a $k$-clique containing $v_{1}$. Since $v_{1}$ was an arbitrary starting point, this algorithm can be repeated for any vertex, and every $v \in V(C)$ appears in a clique of size $k$.

We also observe that while 2 -covers can be disconnected, $k$-covers with $k \geqslant 3$ are connected. Additionally, since the diameter of a graph is the longest shortest path between any two vertices, and every two vertices has a common neighbor, $k$-covers with $k \geqslant 3$ have diameter at most two.

Proposition 17. A graph $G$ is $k$-domination-critical if and only if $\bar{G}$ is a minimal $k$ cover.

Proof. If $G$ is $k$-domination-critical, then $G$ has a dominating set of size $k$, but no dominating set of size $k-1$. Thus, every $(k-1)$-subset of vertices in $\bar{G}$ has a common neighbor, and $\bar{G}$ is a $k$-cover. Furthermore, since $G$ is $k$-domination-critical, for any two non-adjacent vertices $u, v, \gamma(G+(u, v))=k-1$. In other words, if any edge $(u, v)$ is removed from $\bar{G}$, then there is at least one $(k-1)$-subset of vertices that no longer has a common neighbor. Thus, $\bar{G}$ is a minimal $k$-cover.

Conversely, assume that $\bar{G}$ is a minimal $k$-cover. Then, for all $e \in E(\bar{G}), \bar{G}-e$ is not a $k$-cover. This implies that there is some set $D \subseteq V(\bar{G})$ of size $k-1$ that does not have a common neighbor. In other words, for all $v \in(V(\bar{G}) \backslash D), v$ is not adjacent to some vertex in $D$. In other words, $D$ is a dominating set of size $k-1$ in $G$. Thus, we have shown that when any edge is added to $G$ (or removed from $\bar{G}$ ), there is a dominating set of size $k-1$ in $G$. Thus, in order to prove that $G$ is $k$-domination-critical, it remains to show that there exists a dominating set of size $k$ in $G$, and that there does not exist a dominating set of size $k-1$ in $G$. 
Clearly, there is no dominating set of size $k-1$ in $G$, since $\bar{G}$ is a $k$-cover. We will now show that $G$ contains a dominating set of size $k$. Consider any two non-adjacent vertices $u, v \in V(G)$. Without loss of generality, let $D=\left\{i_{1}, \ldots, i_{k-2}, u\right\}$ be the dominating set of size $k-1$ in $G+(u, v)$. Since $D$ is a dominating set in $G+(u, v)$, but not a dominating set in $G$, the only vertex that $D$ does not dominate in $G$ is $v$. Thus, $D+v$ is a dominating set in $G$, and since $|D+v|=k, G$ is $k$-domination-critical.

We note that in [32], Sumner and Blitch extensively explore properties of 3-dominationcritical graphs. They explicitly categorize 2-domination-critical graphs as follows:

Theorem 18 (Sumner and Blitch, 1983). A graph $G$ is 2-domination-critical if and only if $\bar{G}=\cup_{i=1}^{n} K_{1, n_{i}}$ with $n \geqslant 1$.

The Sumner-Blitch categorization of 2-domination-critical graphs is equivalent to Definition 13 , since for any 2-cover, every vertex is adjacent to at least one other vertex.

Example 19. Here we see a 2-cover on 16 vertices. We note that this 2 -cover is the union of two $K_{1,3}$ graphs, two $K_{1,2}$ graphs, and a $K_{1,1}$ graph. Additionally, every single vertex (every set $S$ with $|S|=1$ ) has a common neighbor.

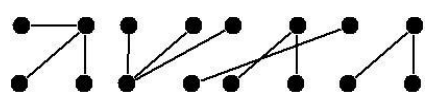

Our categorization is an extension of the Sumner-Blitch categorization since Definition 13 is generalized for the complements of $k$-dominating graphs, although further characterizations are needed for minimal $k$-covers. We now link minimal $k+1$-covers with $k$-dominating graphs.

Theorem 20. A graph $G=(V, E)$ has a dominating set of size $k$ if and only if for all minimal $k+1$-covers $C=\left(V, E_{\text {cov }}\right), E(G) \cap E_{\text {cov }} \neq \emptyset$.

Via this theorem, we characterize graphs with dominating sets of size one in terms of 2-covers. Since one is the smallest dominating set, this explains why we only define $k$-covers for $k \geqslant 2$.

Proof. Assume $G=(V, E)$ has a dominating set $D \subseteq V(G)$ of size $k$. Consider any minimal $k+1$-cover $C=\left(V, E_{\text {cov }}\right)$. Since $D$ is also a subset of $V(C)$, and since $|D|=k$, by Definition $13, D$ has a common neighbor $v$ in $C$. Thus, for every $u \in D,(u, v) \in E_{\text {cov }}$. Since $D$ is a dominating set in $G$ and $v \notin D$, there must exist an edge $(u, v) \in E(G)$ with $u \in D$. Since $(u, v) \in E(G)$ and $(u, v) \in E_{\text {cov }}, E(G) \cap E_{\text {cov }} \neq \emptyset$.

Conversely, assume that for all minimal $k+1$-covers $C=\left(V, E_{\text {cov }}\right), E(G) \cap E_{\text {cov }} \neq \emptyset$. We must show that $G$ contains a dominating set of size $k$. We proceed by contradiction. Assume that $G$ does not contain a dominating set of size $k$, and form a new graph $G_{\text {crit }}$ by adding edges to $G$ until the resulting graph is $k+1$-domination-critical. Thus, $E(G) \subseteq E\left(G_{\text {crit }}\right)$, and $G_{\text {crit }}$ has a dominating set of size $k+1$, but no dominating set of size $k$. 
Next, we consider the complement of $G_{\text {crit }}$ (denoted by $\left.\overline{G_{\text {crit }}}\right)$. Since $G_{\text {crit }}$ is $k+1$ critical, $\overline{G_{\text {crit }}}$ is a minimal $k+1$-cover by Proposition 17 . We note that $E\left(\overline{G_{\text {crit }}}\right) \subseteq E(\bar{G})$, and $E\left(\overline{G_{\text {crit }}}\right) \cap E\left(G_{\text {crit }}\right)=\emptyset$. However, $E(G) \subseteq E\left(G_{\text {crit }}\right)$. Therefore, $E\left(\overline{G_{\text {crit }}}\right) \cap E(G)=\emptyset$. But this is a contradiction, since $\overline{G_{\text {crit }}}$ is a minimal $k+1$-cover, and $E(G) \cap E_{\text {cov }} \neq \emptyset$, for all minimal $k+1$-covers $C=\left(V, E_{\text {cov }}\right)$. Thus, $G$ contains a dominating set of size $k$.

We note that, according to Thm. 20, in order to demonstrate that a graph $G$ does not have dominating set of size $k$, it is sufficient to produce a $k+1$-cover $C$ that is a subgraph of $\bar{G}$. This $k+1$-cover then becomes a certificate of the non-existence of a $k$-dominating set: it is a coNP certificate. However, even though the cover itself is polynomial-size in $|G|$, there are no known polynomial-time algorithms for verifying that every $k$-subset of $C$ has a common neighbor. Therefore, there is no conflict between the use of $k$-covers as certificates, and the conjectured non-equality of NP and coNP.

We now explore properties of $k$-covers.

Theorem 21. A graph $C$ is a $k$-cover if and only if, for all $S \subseteq V(C)$ with $1 \leqslant|S| \leqslant k-1$, there exists a clique $Q$ in $c m N_{C}(S)$ such that $|Q|=k-|S|$. Moreover, every $v \in c m N_{C}(S)$ appears in a clique $Q$ of size $k-|S|$.

Proof. Assume that a graph $C$ is a $k$-cover. Given $S \subseteq V(C)$, if $|S|=k-1$, we know by definition of a $k$-cover that $c m N(S)$ is non-empty. Therefore, a clique $Q$ of size one exists in $\mathrm{cmN}(S)$, and moreover, all vertices in $\mathrm{cmN}(S)$ trivially form cliques of size one.

Now consider $S \subseteq V(C)$ with $1 \leqslant|S| \leqslant k-2$. As before, by definition, since $|S|<k-1, c m N(S)$ is non-empty. Let $v_{1} \in c m N(S)$. Since $\left|S \cup v_{1}\right| \leqslant k-1$, the set $S \cup v_{1}$ has a common neighbor $v_{2}$. Thus, the set $\left\{v_{1}, v_{2}\right\}$ forms a clique of size two in $\operatorname{cmN}(S)$. If $\left|S \cup v_{1} \cup v_{2}\right| \leqslant k-1$, we repeat this operation, and we note that the set $S \cup v_{1} \cup v_{2}$ has a common neighbor $v_{3}$, and that $\left\{v_{1}, v_{2}, v_{3}\right\}$ forms a clique of size three in $c m N(S)$. We repeat this operation exactly $k-|S|-1$ times until we have formed the $k-|S|$-clique $Q=\left\{v_{1}, v_{2}, \ldots, v_{k-|S|}\right\}$ in $\operatorname{cm} N(S)$.

Therefore, for all $1 \leqslant|S| \leqslant k-1$, there exists a clique $Q$ in $\operatorname{cm} N(S)$ such that $|Q|=k-|S|$. Moreover, every $v \in \operatorname{cm} N(S)$ appears in a clique $Q$ of size $k-|S|$, since this operation can be repeated with reference to any vertex $v$.

Conversely, let $C$ be a graph such that for all $S \subseteq V(C)$ with $1 \leqslant|S| \leqslant k-1$, there exists a clique $Q$ in $c m N(S)$ such that $|Q|=k-|S|$. In particular, let $S$ be a subset of $V(C)$ with $|S|=k-1$. Then, there exists a clique $Q$ of size one in $\operatorname{cm} N(S)$. Therefore, by definition, $C$ is a $k$-cover.

We have already shown that every vertex in a $k$-cover appears in a $k$-clique, but we have not discussed how these $k$-cliques intersect. We present the following conjecture.

Conjecture 22. A graph $C$ is a $k$-cover if and only if for all $S \subseteq V(C)$ with $|S|=k-1$, there exists a map $q$ from $v \in S$ to $k$-cliques in $C$ such that for any $u, v \in S,|q(u) \cap q(v)| \geqslant$ $k-2$.

Example 23. Here is an example of Conjecture 22 on a 3-cover of 7 vertices. 


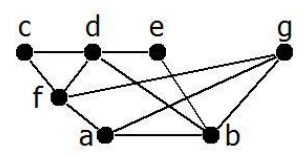

Consider $S=\{c, g\}$. Then, if $q(c)=\{c, d, f\}$ and $q(g)=\{a, f, g\},|q(c) \cup q(g)|=|\{f\}|=$ $1=k-2$. Additionally, consider $S=\{e, g\}$. Then, if $q(e)=\{b, d, e\}$ and $q(g)=\{a, b, g\}$, $|q(e) \cup q(g)|=|\{b\}|=1=k-2$. Upon inspection, we can see that given any set $S$ with $|S|=k-1=2$, a similar map $q$ can be constructed and the conjecture holds.

\section{$4.3 k$-Covers, $k$-Dominating Sets and a Universal Gröbner Basis of $I\left(n, k, n^{\prime}, l, r\right)$}

In Section 4.1, we outlined a representation of the $k$-dominating set problem as a system of polynomial equations, and defined the ideal $I\left(n, k, n^{\prime}, l, r\right)$. In Theorem $9, I\left(n, k, n^{\prime}, l, r\right)$ was defined by three, high-degree polynomials $P_{G}^{k}, P_{H}^{l}$ and $P_{G \square H}^{r}$, and their associated boolean edge equations. Each of these polynomials was a series of products, with each product a sum of products. Thus, the polynomials $P_{G}^{k}, P_{H}^{l}$ and $P_{G \square H}^{r}$ were a brute-force enumeration of every possible vertex set of size $k$, dominating or otherwise, in the graph.

In this section, we present another representation of the ideal $I\left(n, k, n^{\prime}, l, r\right)$. This representation is based on $k+1$-covers, with each polynomial equation corresponding to a minimal $k+1$-cover of $V(G)$. Thus, $I\left(n, k, n^{\prime}, l, r\right)$ can also be generated by many different polynomials of comparatively low degree, as opposed to only three polynomials of high degree. However, there is currently no known algorithm for enumerating minimal $k+1$-covers, which is equivalent to the problem of enumerating $k+1$-domination-critical graphs. Thus, another point of comparison between the representation expressed here and Theorem 9 is that the polynomials described in Theorem 9 can be explicitly written down, whereas polynomials based on $k+1$-covers, while combinatorially explicit, may be difficult to write down explicitly enough for the purpose of computation. However, the potential advantage of the cover representation is that $k+1$-covers are collections of edges that satisfy the linear factor criterion (Definition 5). Therefore, by Theorem 6 and Corollary 8, the representation described below is a universal Gröbner basis.

In presenting a combinatorial interpretation of any Gröbner basis, we must be particularly careful of input instances where the system of polynomial equations is infeasible. In these cases, the associated variety is empty and the associated ideal is the entire ring. This implies that the universal Gröbner basis is simply the number one. In our case, we will exclude input instances where the system of polynomial equations is infeasible by restricting the values of $n, n^{\prime}, k$ and $l$. However, in Theorem 38, we will show that the instances lost by this restriction correspond exactly to instances where Vizing's conjecture is known to be true.

Before we begin, we introduce some notation. Let $\mathscr{C}_{k+1}^{n}$ be the set of all minimal $k+1$-covers on $n$ vertices. For $C \in \mathscr{C}_{k+1}^{n}$, let

$$
P(C):=\prod_{e \in E(C)}(e-1) .
$$

the EleCtronic Journal of COMBinatorics 19(2) (2012), \#P1 
Theorem 24. There is a bijection between the solutions of the following system of equations and the labeled graphs $G$ in $n$ vertices with a dominating set of size $k$.

$$
\begin{aligned}
e_{i j}^{2}-e_{i j} & =0, \quad \text { for } 1 \leqslant i<j \leqslant n, \\
P(C) & =0, \quad \text { for each } C \in \mathscr{C}_{k+1}^{n} .
\end{aligned}
$$

We note that if $k=n$, there are no $n+1$ covers on $n$ vertices. Thus, $\mathscr{C}_{n+1}^{n}=\emptyset$, and the only equations that appear are of the form $e^{2}-e=0$. Thus, any graph is a solution to this system, which is reasonable since every graph has a dominating set of size $n$.

Proof. As in the proof of Theorem 9, we must define a map $\phi$ and show that $\phi$ provides a bijection between the set of solutions and the set of graphs with a dominating set of size $k$. We will use the same map $\phi$ defined in the proof of Theorem 9 , and thus, we already know that the map is one-to-one, and we only need show that the image of $\phi$ is the set of graphs with a dominating set of size $k$, and that the map is onto.

To consider the image of the map $\phi$, we note that, as in Theorem 9, every solution corresponds to a particular graph $G$ (the assignment of the variables $e_{i j}$ to zero or one simply turns the edges "on" or "off" in $G$ ). Since the cover equations (Eqs. 7) are satisfied, this implies that for all minimal $k+1$-covers, $E(G) \cap E(C) \neq \emptyset$. Then, by Theorem 20, $G$ has a dominating set of size $k$.

To show that $\phi$ is onto, consider a graph $G$ in $n$ vertices that has a dominating set of size $k$. As before, we must show that $\phi^{-1}(G)$ (defined by setting variables $e_{i j}$ to zero or one, depending on whether or not $e_{i j} \in E(G)$ ) maps to a solution. Clearly, the boolean equations $e_{i j}^{2}-e_{i j}=0$ are satisfied. Since the graph $G$ has a dominating set of size $k$, for any minimal $k+1$-cover $C, E(G) \cap E(C) \neq \emptyset$ by Theorem 20. Therefore, at least one edge in every cover is "on" and the cover equations (Eqs. 7) are satisfied.

Let $I(n, k)$ denote the ideal generated by polynomials described in Theorem 24 . We will now prove that this representation is a universal Gröbner basis. We note that we discovered the connection between covers and Gröbner bases by experimental investigations using CoCoA Lib [1].

Corollary 25. The basis $I(n, k)$ described in Theorem 24 is a universal Gröbner basis.

Proof. We must show that $\left\{P(C): C \in \mathscr{C}_{k+1}^{n}\right\}$ satisfies the linear factor criterion (Def. $5)$. Let $C_{i}, C_{j}, C_{k}$ be covers in $\mathscr{C}_{k+1}^{n}$. Since each are minimal covers, it is clear that $C_{k}$ can never be a proper subset of $C_{i} \backslash C_{i} \cap C_{j}$ or $C_{i} \backslash C_{i} \cap C_{j}$. From Thm. 6 and Cor. 8, we can see that the basis of $I(n, k)$ described in Thm. 24 is a universal Gröbner basis.

In Section 4.1, we used the system of polynomial equations defined in Theorem 9 to model the larger question of dominating sets in graphs $G, H$ and $G \square H$ (Theorem 11). We will repeat the process here, using the cover-based model from Theorem 24 as a building block. However, a system of polynomial equations based on $k+1$-covers is also intrinsically based on graph intersections. Thus, we must define the edge variables that appear in the intersection of an arbitrary $r$-cover on $n n^{\prime}$ vertices and an arbitrary product graph. 
Definition 26. Given graphs $G$ and $H$, let $C$ be a graph on the vertices $V(G) \times V(H)$. Then the set $\cap_{\square} C \subseteq(E(G) \cup E(H))$ is defined to be the following set of edges:

$$
\cap_{\square} C=\left\{\left(g, g^{\prime}\right):\left(g h, g^{\prime} h\right) \in E(C)\right\} \cup\left\{\left(h, h^{\prime}\right):\left(g h, g h^{\prime}\right) \in E(C)\right\} .
$$

Now we present the cover-based model that links dominating sets in $G, H$ and $G \square H$. As before, this system of polynomial equations will be used in the algebraic representation of Vizing's conjecture in Section 5, and the restriction on the values of $n, k, n^{\prime}, l$ and $r$ will be more meaningful in that context.

Theorem 27. Let $n, k, n^{\prime}, l$ and $r$ be integers such that $r \geqslant \min \left(n, n^{\prime}\right)$. There is a bijection between the set of solutions of the following system of polynomial equations and the set of labeled graphs $G, H$ in $n, n^{\prime}$ vertices with dominating sets of size $k, l$ respectively such that their Cartesian product graph $G \square H$ has a dominating set of size $r$.

Representing a graph $G$ in $n$ vertices with a dominating set of size $k$ :

$$
\begin{aligned}
e_{i j}^{2}-e_{i j} & =0, \quad \text { for } 1 \leqslant i<j \leqslant n, \\
P(C) & =0, \quad \text { for each } C \in \mathscr{C}_{k+1}^{n} .
\end{aligned}
$$

Representing the graph $H$ in $n^{\prime}$ vertices with a dominating set of size l:

$$
\begin{array}{r}
e_{i j}^{\prime 2}-e_{i j}^{\prime}=0, \quad \text { for } 1 \leqslant i<j \leqslant n^{\prime}, \\
P(C)=0, \quad \text { for each } C \in \mathscr{C}_{l+1}^{n^{\prime}} .
\end{array}
$$

Representing the Cartesian product graph $G \square H$ with a dominating set of size $r$ :

$$
P\left(\cap_{\square} C\right)=0, \quad \text { for each } C \in \mathscr{C}_{r+1}^{n n^{\prime}} .
$$

Proof. It is clear from Theorem 24 that there is a bijection between the solutions of the equations representing graphs $G$ and $H$ and the set of labeled graphs in $n, n^{\prime}$ vertices with dominating sets of size $k, l$ respectively. The equation representing $G \square H$ is of the same form, except that it takes into account the unique structure of the product graph.

Assume that $G \square H$ has a dominating set $D \subseteq V(G \square H)$ of size $r$. We will show that the cover equations associated with $G \square H$ are satisfied. Let $C_{r+1}^{n n^{\prime}}$ be a minimal $r+1$-cover on $V(G \square H)$. Since $C_{r+1}^{n n^{\prime}}$ is an $r+1$-cover, every subset of size $r$ has a common neighbor in $V\left(C_{r+1}^{n n^{\prime}}\right)=V(G \square H)$. In particular, $D$ has a common neighbor $g h \in V\left(C_{r+1}^{n n^{\prime}}\right)$. Since $D$ is a dominating set of $G \square H$ and $g h$ is not in $D$, the vertex $g h$ must be dominated by a vertex in $D$. Therefore, either $g h^{\prime} \in D$ with $\left(h, h^{\prime}\right) \in E(H)$, or $g^{\prime} h \in D$ with $\left(g, g^{\prime}\right) \in E(G)$. In first case, the edge $\left(g h^{\prime}, g h\right) \in E\left(C_{r+1}^{n n^{\prime}}\right) \cap E(G \square H)$, and in the second case, the edge $\left(g^{\prime} h, g h\right) \in E\left(C_{r+1}^{n n^{\prime}}\right) \cap E(G \square H)$. In either case, $E\left(C_{r+1}^{n n^{\prime}}\right) \cap E(G \square H) \neq \emptyset$, and each of the cover equations associated with $G \square H$ are satisfied.

Conversely, assume that each of the cover equations associated with $G \square H$ are satisfied. We must show that $G \square H$ has a dominating set of size $r$. We proceed by contradiction. 
Assume that $G \square H$ does not have a dominating set of size $r$, and consider the complement of $G \square H$ (denoted by $\overline{G \square H}$ ).

Since $G \square H$ does not contain a dominating set of size $r$, every subset of size $r$ in $V(\overline{G \square H})$ has a common neighbor. Thus, by Definition $13, \overline{G \square H}$ is a $r+1$-cover. Furthermore, $E(G \square H) \cap E(\overline{G \square H})=\emptyset$. We remove edges from $\overline{G \square H}$ until we find a subgraph $C$ that is a minimal $r+1$-cover. Since $C$ is a subgraph of $\overline{G \square H}, E(G \square H) \cap E(C)=\emptyset$. However, edges in $C$ of the form $\left(g h, g^{\prime} h^{\prime}\right)$ where $g \neq g^{\prime}$ and $h \neq h^{\prime}$ do not correspond to variables in our system of polynomial equations, and thus, we have not yet shown that the set $\cap_{\square} C$ is non-empty and the equation $P\left(\cap_{\square} C\right)=0$ is not satisfied. We must show that $C$ contains at least one edge of the form $\left(g h, g^{\prime} h\right)$ or $\left(g h, g h^{\prime}\right)$.

By assumption, $r \geqslant \min \left(n, n^{\prime}\right)$. Without loss of generality, let $n^{\prime} \geqslant n$ and let $S \subseteq$ $V(C)=V(\overline{G \square H})$ be a subset of size $r$ such that $\{g: g h \in S\}=V(G)$. In other words, choose $S$ such that there is at least one vertex in $S$ per $G$-level. Since $C$ is an $r+1$-cover, $S$ has a common neighbor $g h \in V(C)$, and since $g h$ is connected to every vertex in $S$ and there is at least one vertex per $G$-level in $S$, there exists a vertex $g h^{\prime} \in S$. Thus, $\left(g h, g h^{\prime}\right) \in E(C)$, implying the set $\cap_{\square} C$ is non-empty. But recall that $E(G \square H) \cap E(C)=\emptyset$, which implies that the equation $P(C)=0$ is not satisfied. This is a contradiction with our assumption that each of the cover equations associated with minimal $r+1$-covers are satisfied. Thus, $G \square H$ contains a dominating set of size $r$.

The ideal described by Theorem 27 is the set of polynomials vanishing on graphs $G, H$ with dominating sets $k, l$ respectively, such that $G \square H$ has a dominating set of size $r$. Thus, the ideal described by Theorem 27 is the same as the ideal described by Theorem 11: both are $I\left(n, k, n^{\prime}, l, r\right)$. However, the question of whether the basis described by Theorem 11 is a universal Gröbner basis is not immediately resolvable. Given two $r+1-$ covers $C$ and $C^{\prime}$ on $n n^{\prime}$ vertices, it does seem possible that for large values of $r$ and smaller values of $k$ and $l$, a $k+1$-cover on $n$ vertices (or an $l+1$-cover on $n^{\prime}$ vertices) might be a proper subgraph of $C \cap C^{\prime}$. In such a scenario, the collection of linear factors would not satisfy the linear factor criterion described by Definition 5 , and the basis would not be a universal Gröbner basis. The natural question to then pose is the following: for what values of $r$ is the cover representation of $I\left(n, k, n^{\prime}, l, r\right)$ a universal Gröbner basis? In Section 5, we will show that this question is equivalent to Vizing's conjecture.

\section{An Algebraic Formulation of Vizing's Conjecture}

We now express Vizing's conjecture in terms of the ideals described in Section 4. By defining two particular zero-dimensional, radical ideals and then intersecting them, we transform Vizing's conjecture from a question about dominating sets and product graphs to an ideal membership question involving the product of two polynomials. This algebraic representation suggests a variety of computational approaches, from large-scale, sparse linear algebra computations such as those demonstrated in [24, 23, 26] to Gröbner basis calculations customized for the linear factor criterion (Def. 5). Although Gröbner basis computations and the ideal membership question itself are both known to be EXPSPACE- 
complete [16], algebraic algorithms customized for specific NP-complete combinatorial properties (such as graph 3-colorability) have yielded surprisingly practical computation approaches on some large examples [23]. However, refinements to known algorithms based on the algebraic and combinatorial properties of these particular dominating set representations have not yet been explored. We leave this question for future work.

After describing the algebraic representation of Vizing's conjecture, we transfer the ideal membership question involving the product of two cover polynomials back to the realm of graph theory. This transformation yields a conjecture involving $k$-covers that is the complement of Vizing's conjecture. We conclude by reclaiming a known result with a cover-based proof, and linking universal Gröbner bases to Vizing's conjecture.

\subsection{Ideals, Varieties and Vizing's Conjecture}

We begin by defining three different varieties, and then relating the vanishing ideals associated with these varieties to the ideals described in Section 4. Each of the following varieties are a set of points such that each point represents a pair of graphs $G, H$ in $n, n^{\prime}$ vertices such that $G \square H$ has a dominating set of size $k l-1$. Additionally,

1. Let $V_{l}^{k-1}$ be a variety such that every point represents a pair of labeled graphs $G, H$ with dominating sets of size $k-1, l$ respectively.

2. Let $V_{l-1}^{k}$ be a variety such that every point represents a pair of labeled graphs $G, H$ with dominating sets of size $k, l-1$ respectively.

3. Let $V_{l}^{k}$ be a variety such that every point represents a pair of labeled graphs $G, H$ with dominating sets of size $k, l$ respectively.

Throughout this section, graphs are always labeled graphs. We define the following ideals.

$$
I_{l}^{k-1}:=I\left(n, k-l, n^{\prime}, l, k l-1\right), \quad I_{l-1}^{k}:=I\left(n, k, n^{\prime}, l-l, k l-1\right), \quad I_{l}^{k} \quad:=I\left(n, k, n^{\prime}, l, k l-1\right) .
$$

Notice that in each of these ideals, we have set $r=k l-1$. According to the definitions of $I\left(n, k, n^{\prime}, l, r\right)$ given in Section $4, V_{l}^{k}=V\left(I_{l}^{k}\right), V_{l}^{k-1}=V\left(I_{l}^{k-1}\right)$ and $V_{l-1}^{k}=V\left(I_{l-1}^{k}\right)$. Recall that if a given ideal $I$ is radical, then $I(V(I))=I$. Since the ideals $I_{l}^{k}, I_{l}^{k-1}$ and $I_{l-1}^{k}$ are radical (by Lemma 2), regardless of whether we choose the representation of $I_{l}^{k}$ from Section 4.1, or the representation of $I_{l}^{k}$ from Section 4.3, $I_{l}^{k}=I\left(V_{l}^{k}\right), I_{l}^{k-1}=$ $I\left(V_{l}^{k-1}\right)$ and $I_{l-1}^{k}=I\left(V_{l-1}^{k}\right)$. In the following lemmas, we will relate Vizing's conjecture to these ideals and varieties. It is important to note that the algebraic representation of Vizing's conjecture described below is independent of the internal representation of the ideal $I\left(n, k, n^{\prime}, l, r\right)$. In other words, the following lemmas will hold for any representation of $I_{l}^{k}, I_{l}^{k-1}$ and $I_{l-1}^{k}$ as long as the ideals are radical, and $I_{l}^{k}=I\left(V_{l}^{k}\right), I_{l}^{k-1}=I\left(V_{l}^{k-1}\right)$ and $I_{l-1}^{k}=I\left(V_{l-1}^{k}\right)$. Thus, if another representation of $I\left(n, k, n^{\prime}, l, r\right)$ is found in the future with a differing set of computational or combinatorial properties, this formulation of Vizing's conjecture remains valid.

Lemma 28. $V_{l}^{k-1} \cup V_{l-1}^{k}=V_{l}^{k}$ if and only if Vizing's conjecture is true. 
Proof. Every point in $V_{l}^{k}$ corresponds to a graph $G$ with $\gamma(G) \leqslant k$ and a graph $H$ with $\gamma(H) \leqslant l$ such that $\gamma(G \square H) \leqslant k l-1$.

Assume that Vizing's conjecture is true. Thus, $\gamma(G) \gamma(H) \leqslant \gamma(G \square H)$ for all graphs $G, H$. In particular, if $\gamma(G)=k$ and $\gamma(H)=l$, then $k l \leqslant \gamma(G \square H)$. In particular, $\gamma(G \square H)$ can never equal $k l-1$. Thus, if there is a pair of graphs $G, H$ such that $\gamma(G \square H) \leqslant k l-1$, then either $\gamma(G) \leqslant k-1$ or $\gamma(H) \leqslant l-1$. Thus, any point appearing in $V_{l}^{k}$ also appears in $V_{l}^{k-1} \cup V_{l-1}^{k}$, and $V_{l}^{k-1} \cup V_{l-1}^{k}=V_{l}^{k}$.

Conversely, assume that $V_{l}^{k-1} \cup V_{l-1}^{k}=V_{l}^{k}$. Then, for every pair of graphs $G, H$ such that $\gamma(G \square H) \leqslant k l-1$, then either $\gamma(G) \leqslant k-1$ or $\gamma(H) \leqslant l-1$. In other words, if $\gamma(G)=k$ and $\gamma(H)=l$, then $\gamma(G \square H)>k l-1$. This implies that $\gamma(G) \gamma(H) \leqslant \gamma(G \square H)$, or that Vizing's conjecture is true.

Lemma 29. $I_{l}^{k-1} \cap I_{l-1}^{k}=I_{l}^{k}$ if and only if Vizing's conjecture is true.

Proof. If $I_{l}^{k-1} \cap I_{l-1}^{k}=I_{l}^{k}$, then $V_{l}^{k-1} \cup V_{l-1}^{k}=V_{l}^{k}$ (and vice versa). This can be seen via the results in Section 2, since if $I_{l}^{k-1} \cap I_{l-1}^{k}=I_{l}^{k}$, then $V\left(I_{l}^{k-1} \cap I_{l-1}^{k}\right)=V\left(I_{l}^{k-1}\right) \cup V\left(I_{l-1}^{k}\right)=$ $V_{l}^{k-1} \cup V_{l-1}^{k}=V\left(I_{l}^{k}\right)=V_{l}^{k}$. Thus, Vizing's conjecture is true via Lemma 28.

We have now equated Vizing's conjecture with a question about the equality of two ideals. To prove this equality, we must prove the inclusion in both directions. The inclusion in one direction is fairly easy to see; however, the inclusion in the other direction remains open.

Lemma 30. $V_{l}^{k-1} \cup V_{l-1}^{k} \subseteq V_{l}^{k}$.

Proof. Consider any point $p$ in $V_{l}^{k-1} \cup V_{l-1}^{k}$. This point either represents graphs $G, H$ with dominating sets $k-1, l$ respectively, or graphs $G, H$ with dominating sets $k, l-1$ respectively (in both cases, $G \square H$ contains a dominating set of size $k l-1$ ). However, if $G$ contains a dominating set of size $k-1$ (or $l-1$ ), $G$ also contains a dominating set of size $k$ (or size $l$ ). Therefore, any point representing graphs $G, H$ with dominating sets $k-1, l$ respectively, or graphs $G, H$ with dominating sets $k, l-1$ respectively also represents graphs $G, H$ with dominating sets $k, l$ respectively. Thus, any point $p \in V_{l}^{k-1} \cup V_{l-1}^{k}$ is also a point $p \in V_{l}^{k}$.

Corollary 31. $I_{l}^{k} \subseteq I_{l}^{k-1} \cap I_{l-1}^{k}$.

Proof. Since $V_{l}^{k-1} \cup V_{l-1}^{k} \subseteq V_{l}^{k}$, this implies that $I\left(V_{l}^{k}\right) \subseteq I\left(V_{l}^{k-1} \cup V_{l-1}^{k}\right)$, which implies that $I_{l}^{k} \subseteq I_{l}^{k-1} \cap I_{l-1}^{k}$ (see [11], Chapter 4).

Thus, in order to conclude a proof Vizing's conjecture, we must show that $I_{l}^{k-1} \cap I_{l-1}^{k} \subseteq$ $I_{l}^{k}$. However, recall from the conclusion of Section 2, if the representations of $I_{l}^{k}, I_{l}^{k-1}$ and $I_{l-1}^{k}$ satisfy the conditions specified in Lemma 3, we can explicitly describe a basis for $I_{l}^{k-1} \cap I_{l-1}^{k}$ :

$$
I_{l}^{k-1} \cap I_{l-1}^{k}=\sqrt{I_{l}^{k-1} \cdot I_{l-1}^{k}}=\left\langle f g: f \in I_{l}^{k-1}, g \in I_{l-1}^{k}\right\rangle+\left\langle e^{2}-e\right\rangle .
$$


Thus far in this section, without specifying the representation for $I_{l}^{k}$, etc., we have shown that proving Vizing's conjecture is equivalent to proving the equality of two ideals. We will now show that for each of the representations specified in Section 4, proving the opposite inclusion reduces to a problem in ideal membership, which implies, far more significantly, that proving Vizing's conjecture reduces to a computational problem in Gröbner bases or linear algebra.

\section{Using the Representation from Theorem 11:}

Let $h$ be any polynomial in $I_{l}^{k-1} \cap I_{l-1}^{k}$, where $I_{l}^{k-1}$ and $I_{l-1}^{k}$ are represented using the polynomials defined by Theorem 9 . Then $h \in I_{l}^{k-1} \cap I_{l-1}^{k}$ can be written as:

$$
\begin{aligned}
h= & (\cdot) P_{G}^{k-1} P_{G}^{k}+(\cdot) P_{G}^{k-1} P_{H}^{l-1}+(\cdot) P_{G}^{k-1} P_{G \square H}+(\cdot) P_{G}^{k-1}\left(e^{2}-e\right)+(\cdot) P_{H}^{l} P_{G}^{k}+(\cdot) P_{H}^{l} P_{H}^{l-1} \\
& +(\cdot) P_{H}^{l} P_{G \square H}+(\cdot) P_{H}^{l}\left(e^{2}-e\right)+(\cdot) P_{G \square H} P_{G}^{k}+(\cdot) P_{G \square H} P_{H}^{l-1}+(\cdot) P_{G \square H} P_{G \square H} \\
& +(\cdot) P_{G \square H}\left(e^{2}-e\right)+(\cdot)\left(e^{2}-e\right) P_{G}^{k}+(\cdot)\left(e^{2}-e\right) P_{H}^{l-1} \\
& +(\cdot)\left(e^{2}-e\right) P_{G \square H}+(\cdot)\left(e^{2}-e\right)\left(e^{2}-e\right)+(\cdot)\left(e^{2}-e\right),
\end{aligned}
$$

where $P_{G \square H}$ is equal to $P_{G \square H}^{k l-1}$. We must show that $h$ is also in $I_{l}^{k}$. Specifically, we must show that there exists polynomial coefficients such that

$$
h=(\cdot) P_{G}^{k}+(\cdot) P_{H}^{l}+(\cdot) P_{G \square H}+(\cdot)\left(e^{2}-e\right) .
$$

Comparing Eqs. 8 and 9, we see that the only term which is not already expressed in terms of polynomials in $I_{l}^{k}$ is the product $P_{G}^{k-1} P_{H}^{l-1}$. Thus, we must find an algebraic relationship or a syzygy such that

$$
P_{G}^{k-1} P_{H}^{l-1}=(\cdot) P_{G}^{k}+(\cdot) P_{H}^{l}+(\cdot) P_{G \square H}+(\cdot)\left(e^{2}-e\right) .
$$

This is equivalent to asking whether or not $P_{G}^{k-1} P_{H}^{l-1} \in I_{l}^{k}$. Here is the link between Vizing's conjecture and ideal membership: a conjecture about dominating sets in product graphs is an ideal membership question about the product of two polynomials.

Lemma 32. $P_{G}^{k-1} P_{H}^{l-1} \in I_{l}^{k}$ if and only if Vizing's conjecture is true.

Proof. If $P_{G}^{k-1} P_{H}^{l-1} \in I_{l}^{k}$, then any polynomial $h \in I_{l}^{k-1} \cap I_{l-1}^{k}$ is also in $I_{l}^{k}$. In other words, $I_{l}^{k-1} \cap I_{l-1}^{k} \subseteq I_{l}^{k}$. Lemma 30 and Corollary 31 establish the other direction of the inclusion, and $I_{l}^{k-1} \cap I_{l-1}^{k}=I_{l}^{k}$. Thus, by Lemma 29, Vizing's conjecture is true. Conversely, if Vizing's conjecture is true, then $I_{l}^{k-1} \cap I_{l-1}^{k}=I_{l}^{k}$, which implies that $P_{G}^{k-1} P_{H}^{l-1} \in I_{l}^{k}$.

Unfortunately, performing these computations was quite difficult. We worked on machines with dual Opteron nodes, $2 \mathrm{GHz}$ clock speed, and $12 \mathrm{~GB}$ of RAM. We used the custom C++ exact arithmetic linear algebra solver and the method described in [24], [23] and [26]. We first tested the smallest possible example of $n=k=n^{\prime}=l=2$. In order to find the syzygy defined by Eq. 10, we constructed a $100 \times 84$ linear system, solved in under a second, yielding a syzygy of degree 6 . However, when we tested the next largest 
example, with $n=3, k=n^{\prime}=l=2$, we were unable to find a syzygy, although we determined that the syzygy had degree 8 or larger. The degree 7 linear algebra system had size $158,412 \times 2,310$ and took 6,862 seconds $(\approx 2$ hours $)$ to solve. However, the degree 8 linear algebra system remained unsolved even after days of computation, most likely due to the magnitude of the numbers involved. For example, one of the coefficients in $P_{G}^{k}$ was $2,106,048,060$. It is easy to imagine the computationally intensive nature of exact arithmetic on numbers of that order in a linear system with millions of rows and columns. However, we again note that the specific combinatorial properties of these representations have not yet been exploited. It is a question of future work to improve these computations.

\section{Using the Representation from Theorem 27:}

In Lemma 32, we showed that proving Vizing's conjecture using the representation described by Theorem 11 was equivalent to proving that $P_{G}^{k-1} P_{H}^{l-1} \in I_{l}^{k}$. We now show that when the representation of $I_{l}^{k}$ corresponding to covers (Theorem 27) is used, Vizing's conjecture reduces to both an ideal membership question involving the product of two cover polynomials, and a graph theoretic question that is the complement of Vizing's conjecture.

In the cover representation of $I_{l}^{k}$, we denote the various cover polynomials as $P\left(C_{k}^{n}\right), P\left(C_{l}^{n^{\prime}}\right)$ and $P\left(\cap_{\square} C_{k l}^{n n^{\prime}}\right)$. As before, $h \in I_{l}^{k-1} \cap I_{l-1}^{k}$ can be written as follows:

$$
\begin{aligned}
h= & (\cdot) P\left(C_{k}^{n}\right) P\left(C_{k+1}^{n}\right)+(\cdot) P\left(C_{k}^{n}\right) P\left(C_{l}^{n^{\prime}}\right)+(\cdot) P\left(C_{k}^{n}\right) P\left(\cap \square C_{k l}^{n n^{\prime}}\right)+(\cdot) P\left(C_{k}^{n}\right)\left(e^{2}-e\right) \\
& +(\cdot) P\left(C_{l+1}^{n^{\prime}}\right) P\left(C_{k+1}^{n}\right)+(\cdot) P\left(C_{l+1}^{n^{\prime}}\right) P\left(C_{l}^{n^{\prime}}\right)+(\cdot) P\left(C_{l+1}^{n^{\prime}}\right) P\left(\cap \square C_{k l}^{n n^{\prime}}\right)+(\cdot) P\left(C_{l+1}^{n^{\prime}}\right)\left(e^{2}-e\right) \\
& +(\cdot) P\left(\cap \square C_{k l}^{n n^{\prime}}\right) P\left(C_{k+1}^{n}\right)+(\cdot) P\left(\cap \square C_{k l}^{n n^{\prime}}\right) P\left(C_{l}^{n^{\prime}}\right)+(\cdot) P\left(\cap \square C_{k l}^{n n^{\prime}}\right) P\left(\cap \square C_{k l}^{n n^{\prime}}\right) \\
& +(\cdot) P\left(\cap \square C_{k l}^{n n^{\prime}}\right)\left(e^{2}-e\right)+(\cdot)\left(e^{2}-e\right) P\left(C_{k+1}^{n}\right)+(\cdot)\left(e^{2}-e\right) P\left(C_{l}^{n^{\prime}}\right) \\
& +(\cdot)\left(e^{2}-e\right) P\left(\cap \square C_{k l}^{n n^{\prime}}\right)+(\cdot)\left(e^{2}-e\right)\left(e^{2}-e\right)+(\cdot)\left(e^{2}-e\right) .
\end{aligned}
$$

Again, to conclude the proof of Vizing's conjecture, we must show that $h \in I_{l}^{k}$. More specifically, we must show that there exist coefficients such that

$$
h=(\cdot) P\left(C_{k+1}^{n}\right)+(\cdot) P\left(C_{l+1}^{n^{\prime}}\right)+(\cdot) P\left(\cap_{\square} C_{k l}^{n n^{\prime}}\right)+(\cdot)\left(e^{2}-e\right) .
$$

Comparing Eqs. 11 and 12, we see that the only term which is not already expressed in terms of polynomials in $I_{l}^{k}$ is the product $P\left(C_{k}^{n}\right) P\left(C_{l}^{n^{\prime}}\right)$. Thus, we must find an algebraic relationship or a syzygy such that

$$
P\left(C_{k}^{n}\right) P\left(C_{l}^{n^{\prime}}\right)=(\cdot) P\left(C_{k+1}^{n}\right)+(\cdot) P\left(C_{l+1}^{n^{\prime}}\right)+(\cdot) P\left(\cap_{\square} C_{k l}^{n n^{\prime}}\right)+(\cdot)\left(e^{2}-e\right) .
$$

This is equivalent to asking whether or not $P\left(C_{k}^{n}\right) P\left(C_{l}^{n^{\prime}}\right) \in I_{l}^{k}$. However, recall that the cover representations are not just a basis, but a universal Gröbner basis. Our experimental investigations using CoCoA Lib [1] have indicated that that not only is $P\left(C_{k}^{n}\right) P\left(C_{l}^{n^{\prime}}\right) \in I_{l}^{k}$, but that there exists a $P\left(\cap_{\square} C_{k l}^{n n^{\prime}}\right)$ such that $P\left(C_{k}^{n}\right) P\left(C_{l}^{n^{\prime}}\right)=P\left(\cap_{\square} C_{k l}^{n n^{\prime}}\right)$. In other words, $P\left(C_{k}^{n}\right) P\left(C_{l}^{n^{\prime}}\right)$ is itself an element of the Gröbner basis of $I_{l}^{k}$. We were not able to verify 
this conjecture on large examples because of the exponential number of monomials in the expanded cover polynomials. For example, $P\left(C_{k}^{n}\right)$ may be compactly represented as a product of $L$ linear factors, but when $P\left(C_{k}^{n}\right)$ is expanded during the calculation of the Gröbner basis, $2^{L}$ monomials are generated. Indeed, even very small examples often took days of computation. We are interested in exploring modifications to Gröbner basis algorithms to exploit the factored form of these input bases for future work.

We will now prove several lemmas and extrapolate the conjecture that there exists a polynomial $P\left(\cap_{\square} C_{k l}^{n n^{\prime}}\right)$ such that $P\left(C_{k}^{n}\right) P\left(C_{l}^{n^{\prime}}\right)=P\left(\cap_{\square} C_{k l}^{n n^{\prime}}\right)$ to a very specific graph theory conjecture. Recall that $\cap_{\square} C_{k l}^{n n^{\prime}}$ (Definition 26) denotes a specific set of edges.

Lemma 33. Given a minimal $k$-cover $C_{k}^{n}$ and a minimal l-cover $C_{l}^{n^{\prime}}$, if there exists a (kl)-cover $C_{k l}^{n n^{\prime}}$ such that $E\left(C_{k}^{n} \square C_{l}^{n^{\prime}}\right)=\cap_{\square} C_{k l}^{n n^{\prime}}$, then Vizing's conjecture is true.

Proof. If there exists a $(k l)$-cover $C_{k l}^{n n^{\prime}}$ such that $E\left(C_{k}^{n} \square C_{l}^{n^{\prime}}\right)=\cap_{\square} C_{k l}^{n n^{\prime}}$, then $P\left(C_{k}^{n}\right) P\left(C_{l}^{n^{\prime}}\right)=P\left(\cap_{\square} C_{k l}^{n n^{\prime}}\right)$, and $P\left(C_{k}^{n}\right) P\left(C_{l}^{n^{\prime}}\right) \in I_{l}^{k}$. Thus, $I_{l}^{k-1} \cap I_{l-1}^{k}=I_{l}^{k}$, and Vizing's conjecture is true.

We will now define a product graph that specifically relates to the $\cap_{\square}$ intersection.

Definition 34. Given graphs $G$ and $H$, the star product $G \star H$ has vertex set $V(G) \times$ $V(H)$ and edge set $E(G \star H)=E(G \square H) \cup\left\{\left(g h, g^{\prime} h^{\prime}\right): g \neq g^{\prime}\right.$ and $\left.h \neq h^{\prime}\right\}$.

Proposition 35. Given a k-cover $C_{k}^{n}$ and a l-cover $C_{l}^{n^{\prime}}$, then $E\left(C_{k}^{n} \square C_{l}^{n^{\prime}}\right)=$ $\cap_{\square}\left(C_{k}^{n} \star C_{l}^{n^{\prime}}\right)$.

Proof. This follows directly from Definitions 26 and 34.

We note that $C=C_{k}^{n} \star C_{l}^{n^{\prime}}$ contains the largest amount of edges such that $\cap_{\square} C=$ $C_{k}^{n} \square C_{l}^{n^{\prime}}$. The question that remains is the following.

Conjecture 36. Given minimal $k, l$-covers $C_{k}^{n}, C_{l}^{n^{\prime}}, C_{k}^{n} \star C_{l}^{n^{\prime}}$ is a $k l$-cover.

This is the complement of Vizing's conjecture. We observe that we can easily prove two of the known properties of covers on $C_{k}^{n} \star C_{l}^{n^{\prime}}$.

Proposition 37. Given minimal $k, l$-covers $C_{k}^{n}, C_{l}^{n^{\prime}}$, every vertex in $V\left(C_{k}^{n} \star C_{l}^{n^{\prime}}\right)$ is contained in a kl-clique.

Proof. Let $g h$ be a vertex in $C_{k}^{n} \star C_{l}^{n^{\prime}}$. We must show that $g h$ appears in a $k l$-clique. Since $C_{k}^{n}, C_{l}^{n^{\prime}}$ are a $k, l$-covers respectively, $g$ appears in a $k$-clique in $C_{k}^{n}$, and $h$ appears in an $l$-clique in $C_{l}^{n^{\prime}}$. Let $g \cup\left\{g_{1}, \ldots, g_{k-1}\right\}$ be the $k$-clique in $C_{k}^{n}$, and let $h \cup\left\{h_{1}, \ldots, h_{l-1}\right\}$ be the $l$-clique in $C_{l}^{n^{\prime}}$. We claim

$$
Q=\left(g \cup\left\{g_{1}, \ldots, g_{k-1}\right\}\right) \times\left(h \cup\left\{h_{1}, \ldots, h_{l-1}\right\}\right)
$$

is a $k l$-clique in $C_{k}^{n} \star C_{l}^{n^{\prime}}$. Let $g h$ and $g^{\prime} h^{\prime}$ be two vertices in $Q$. If $g=g^{\prime}$, then $\left(h, h^{\prime}\right) \in C_{l}^{n^{\prime}}$, since $h, h^{\prime}$ appear in an $l$-clique. If $h=h^{\prime}$, then $\left(g, g^{\prime}\right) \in C_{k}^{n}$, since $g, g^{\prime}$ appear in an $k$ clique. In both cases, $\left(g h, g^{\prime} h^{\prime}\right)$ is an edge in $C_{k}^{n} \star C_{l}^{n^{\prime}}$. Finally, if $g \neq g^{\prime}$ and $h \neq h^{\prime}$, then $\left(g h, g^{\prime} h^{\prime}\right)$ is an edge in $C_{k}^{n} \star C_{l}^{n^{\prime}}$ by the definition of the star product. Thus, we have shown that $Q$ is a $k l$-clique. 
Additionally, we can see that if $k$ and $l$ are strictly greater than two, then $C_{k}^{n} \star C_{l}^{n^{\prime}}$ has diameter at most two. This can be seen by choosing any two vertices $g h, g^{\prime} h^{\prime}$ in $C_{k}^{n} \star C_{l}^{n^{\prime}}$, and noting that there must exist a third vertex $g^{\prime \prime} h^{\prime \prime}$ such that $g^{\prime \prime} h^{\prime \prime}$ is adjacent to both $g h$ and $g^{\prime} h^{\prime}$. Thus, under these conditions, the diameter of $C_{k}^{n} \star C_{l}^{n^{\prime}}$ is at most two.

When framing Vizing's conjecture in terms of covers and the star product, we can easily reclaim the complement of a known result by El-Zahar and Pareek [12].

Theorem 38. Given minimal $k, l$-covers $C_{k}^{n}, C_{l}^{n^{\prime}}$, such that $k l-1<\min \left(n, n^{\prime}\right)$, then $C_{k}^{n} \star C_{l}^{n^{\prime}}$ is a kl-cover.

Proof. Consider any set $S$ of $k l-1$ vertices in $C_{k}^{n} \star C_{l}^{n^{\prime}}$. Let $P_{k}^{n}$ denote $C_{k}^{n}$-projection of $S$ (the set of $g$ coordinates such that $g h$ is a vertex in $S$ ), and let $P_{l}^{n^{\prime}}$ denote $C_{l}^{n^{\prime}}$-projection of $S$ (the set of $h$ coordinates such that $g h$ is a vertex in $S$ ). Since $k l-1<\min \left(n, n^{\prime}\right)$, $\left|P_{k}^{n}\right|,\left|P_{l}^{n^{\prime}}\right|<\min \left(n, n^{\prime}\right)$. Thus, there exists a vertex $g^{\prime} h^{\prime}$ such that $g \neq g^{\prime}$ and $h \neq h^{\prime}$. Thus, $g h$ is adjacent to $g^{\prime} h^{\prime}$, and $g^{\prime} h^{\prime}$ is the common neighbor of $S$. Since $S$ was a general set, $C_{k}^{n} \star C_{l}^{n^{\prime}}$ is a $k l$-cover.

Since we have shown that $C_{k}^{n} \star C_{l}^{n^{\prime}}$ is a $k l$-cover, by Lemma 33, we have shown that Vizing's conjecture holds on this class of graphs. This is the complement of the result proven in [12]. The restriction placed on the value of $r$ in Theorem 27 now becomes clear. We only define the cover representation of $I_{l}^{k}$ when $k l-l \geqslant \min \left(n, n^{\prime}\right)$. But since Vizing's conjecture holds whenever $k l-1<\min \left(n, n^{\prime}\right)$, the method of intersecting ideals is defined only on the as-yet unproven cases of Vizing's conjecture.

We conclude by drawing a parallel between universal Gröbner bases and Vizing's conjecture based on our experimental investigations with CoCoA Lib [1]. Since a $k+1$ cover is not a proper subset of a $k$-cover, and an $l+1$-cover is not a proper subset of a $l$-cover, if any minimal $k l$-cover can be written as $C_{k}^{n} \star C_{l}^{n^{\prime}}$ for some minimal $C_{k}^{n}, C_{l}^{n^{\prime}}$, then not only is Vizing's conjecture true, but replacing

$$
P\left(\cap_{\square} C\right)=0, \quad \text { for each } C \in \mathscr{C}_{k l}^{n n^{\prime}},
$$

with

$$
P\left(C_{k}^{n} \star C_{l}^{n^{\prime}}\right)=0, \quad \text { for each } C_{k}^{n} \in \mathscr{C}_{k}^{n} \text { and each } C_{l}^{n^{\prime}} \in \mathscr{C}_{l}^{n^{\prime}},
$$

in Theorem 27 would yield a universal Gröbner basis for $I\left(n, k, n^{\prime}, l, r=k l-1\right)$.

\section{Conclusion}

In this paper, we approach a canonical NP-complete problem (dominating set) by modeling it as a system of polynomial equations, reducing the model to its universal Gröbner basis, and then translating the model back to a different system of polynomial equations where the polynomials match one-to-one with domination-critical graphs. This easy translation between polynomials and subgraphs demonstrates the possibilities opened by 
approaching a combinatorial problem via algebraic geometry. In particular, given a problem such as Vizing's conjecture, creating an algebraic formulation is an opportunity for an entirely new set of tools to try their strength against an old open problem. For example, during the actual computation of the universal Gröbner basis, given that both the initial ideal representation and the final universal Gröbner basis have known combinatorial interpretations, is there a graph-theoretic significance to the intermediate polynomials? Do Buchberger's S-pair polynomials correspond to a particular subgraph operation? Since no graph-theoretic counterexample to Vizing's conjecture has been found in 50 years, is it possible that the search for a polynomial counterexample (or proof) is a less prohibitive challenge?

Additionally, the computational significance of the linear factor criterion introduced in this paper is yet to be explored. In this case, the factored form of the both the input and output ideal is known before the computation even begins. With this combinatorial knowledge in hand, could a Gröbner basis algorithm be specifically tailored to avoid certain types of polynomial expansions when computing on ideals of this form? Finally, a universal Gröbner basis that is the product of linear factors is not unique to the dominating set problem; preliminary investigations indicate that other polynomial models of other combinatorial problems (such as independent set and factoring) share this property.

In short, this paper explores algebraic representations of the dominating set problem, focusing on a combinatorial interpretation of the universal Gröbner basis. The consequence of this result on computation is the subject of future work.

\section{Acknowledgements}

The authors would like to thank the anonymous referees for their comments. We also acknowledge the support of NSF DMS-0729251, NSF-CMMI-0926618, DMS-0240058 and the Rice University VIGRE program.

\section{References}

[1] J. Abbott and A.M. Bigatti. CoCoALib: a C++ library for doing Computations in Commutative Algebra. Available at http://cocoa.dima.unige.it/cocoalib.

[2] N. Alon. Combinatorial Nullstellensatz. Combinatorics, Prob. and Computing, 8:729, 1999.

[3] N. Alon and M. Tarsi. Colorings and orientations of graphs. Combinatorica, 12:125134, 1992.

[4] E. Babson, S. Onn, and R. Thomas. The Hilbert zonotope and a polynomial time algorithm for universal Görbner bases. Advances in Applied Mathematics, 30(3):529$544,2003$.

[5] A. Barcalkin and L. German. The external stability number of the cartesian product of graphs. Bul. Akad. Stiinte RSS Moldoven., 1(94):5-8, 1979. 
[6] V. Blanco and J. Puerto. Partial Gröbner bases for multiobjective integer linear optimization. SIAM Journal Discret. Math., 23(2):571-595, 2009.

[7] B.Stjan, B.Brešar, P.Dorbec, W.Goddard, B.Hartnell, M.Henning, S.Klavžar, and D.Rall. Vizing's conjecture: A survey and recent results, 2009. preprint.

[8] B. Buchberger. An algorithm for finding the basis elements of the residue class ring of a zero-dimensional polynomial ideal. Journal of Symbolic Computation, 4(3-4):475$511,2006$.

[9] W. Clark and S. Suen. An inequality related to Vizing's conjecture. Electronic Journal of Combinatorics, 7(Note 4), 2000.

[10] P. Conti and C. Traverso. Buchberger algorithm and integer programming. In Proceedings of the 9th International Symposium, on Applied Algebra, Algebraic Algorithms and Error-Correcting Codes, AAECC-9, pages 130-139. Springer-Verlag, 1991.

[11] D. Cox, J. Little, and D. O'Shea. Ideals, Varieties and Algorithms. Springer, New York, 1998.

[12] M. El-Zahar and C.M. Pareek. Domination number of products of graphs. Ars Combin., 31:223-227, 1991.

[13] S. Eliahou. An algebraic criterion for a graph to be four-colourable. Aportaciones Matemáticas, 6:3-27, 1992.

[14] K.G. Fischer. Symmetric polynomials and Hall's theorem. Discrete Math, 69(3):225234, 1988.

[15] M. Garey and D. Johnson. Computers and Intractability: A Guide to the Theory of NP-Completeness. W.H. Freeman and Company, 1979.

[16] J. Gathen and J. Gerhard. Modern Computer Algebra. Cambridge University Press, Cambridge, second edition, 1999.

[17] B. Hartnell and D. Rall. Domination in cartesian products: Vizing's conjecture. In Domination in graphs, pages 163-189, New York, 1998. Mono. Textbooks Pure and Appl. Math, Dekker.

[18] C.J. Hillar and T. Windfeldt. An algebraic characterization of uniquely vertex colorable graphs. Journal of Combinatorial Theory Series B, 98:400-414, 2008.

[19] M. Kreuzer and L. Robbiano. Computational Commutative Algebra I. SpringerVerlag, 2000.

[20] S. Li and W. Li. Independence number of graphs and generators of ideals. Combinatorica, 1:55-61, 1981.

[21] J.A. De Loera. Gröbner bases and graph colorings. Beitrage zur Algebra und Geometrie, 36(1):89-96, 1995.

[22] J.A. De Loera, C. Hillar, P.N. Malkin, and M. Omar. Recognizing graph theoretic properties with polynomial ideals. Elect. J. of Combinatorics., 17(1):R114, 2010. 
[23] J.A. De Loera, J. Lee, P.N. Malkin, and S. Margulies. Hilbert's Nullstellensatz and an algorithm for proving combinatorial infeasibility. In Proceedings of the twentyfirst international symposium on Symbolic and algebraic computation, pages 197-206. ISSAC, 2008.

[24] J.A. De Loera, J. Lee, S. Margulies, and S. Onn. Expressing combinatorial optimization problems by systems of polynomial equations and the Nullstellensatz. Combinatorics, Probability and Computing, 18(4):551-582, 2009.

[25] L. Lovász. Stable sets and polynomials. Discrete Mathematics, 124:137-153, 1994.

[26] S. Margulies. Computer Algebra, Combinatorics, and Complexity: Hilbert's Nullstellensatz and NP-Complete Problems. University of California at Davis, Ph.D. Thesis, 2008.

[27] Y. Matiyasevich. A criteria for colorability of vertices stated in terms of edge orientations (in russian). Discrete Analysis (Novosibirsk), 26:65-71, 1974.

[28] Y. Matiyasevich. Some algebraic methods for calculation of the number of colorings of a graph (in russian). Zapiski Nauchnykh Seminarov POMI, 293:193-205, 2001.

[29] K. Mizuno and S. Nishihara. Nowhere-zero flow polynomials. Journal of Combinatorial Theory, Series A, 108(2):205-215, 2004.

[30] M. Mnuk. Representing graph properties by polynomial ideals. In Computer Algebra in Scientific Computing, pages 431-444. 4th Inter. Work. on Comp. Alg. in Sci. Comp., 2001.

[31] A. Simis, W. Vasconcelos, and R. Villarreal. On the ideal theory of graphs. Journal of Algebra, 167(2):389-416, 1994.

[32] D. Sumner and P. Blitch. Domination critical graphs. Journal of Cominatorial Theory, Series B, 34:65-76, 1983.

[33] L. Sun. A result on Vizing's conjecture. Discrete Mathematics, 275(1):363-366, 2004.

[34] R. Thomas. A geometric buchberger algorithm for integer programming. Mathematics of Operations Research, 20(4):864-884, 1995.

[35] V. Vizing. Some unsolved problems in graph theory. Uspekhi Mat. Nauk, 23:117-134, 1968. 